\title{
A NOVEL METHOD FOR HANDLING PRE-EXISTING CONDITIONS IN PREDICTION MODELS FOR COVID-19 DEATH
}

\author{
Glen H. Murata, MD* \\ Allison E. Murata, MSIM^ \\ Heather M. Campbell, PharmD, $\mathrm{PhD}^{\wedge}$ \& \\ Benjamin H. Mcmahon, $\mathrm{PhD}^{\%}$ \\ Jenny T. Mao, MD, FCCP ${ }^{\star \#}$
}

* New Mexico VA Health Care System, Albuquerque NM

$\wedge$ VA Cooperative Studies Program - Clinical Research Pharmacy Coordinating Center, Albuquerque NM

\& University of New Mexico College of Pharmacy, Albuquerque NM

\% Los Alamos National Laboratory, Los Alamos NM

\# University of New Mexico School of Medicine, Albuquerque NM 
medRxiv preprint doi: https://doi.org/10.1101/2022.01.22.22269694; this version posted February 21, 2022. The copyright holder for this preprint

(which was not certified by peer review) is the author/funder, who has granted medRxiv a license to display the preprint in perpetuity.

This article is a US Government work. It is not subject to copyright under 17 USC 105 and is also made available for use under a CCO license.

COMORBIDITIES IN MODELS FOR COVID-19 DEATH

\section{ABSTRACT}

Objective: To derive a predicted probability of death (PDeathDx) based upon complete sets of ICD-10 codes assigned to patients prior to their diagnosis of COVID-19. PDeathDx is intended for use as a summary metric for pre-existing conditions in multivariate models for COVID-19 death.

Methods: Cases were identified through the COVID-19 Shared Data Resource (CSDR) of the Department of Veterans Affairs. The diagnosis required at least one positive nucleic acid amplification test (NAAT). The primary outcome was death within 60 days of the first positive test. We retrieved all diagnoses entered into the electronic medical record for visits, on problem lists, and at the time of hospital discharge if they were at least 14 days prior to the NAAT. ICD-9 codes were converted to ICD-10 equivalents using a crosswalk provided by the Centers for Medicare/Medicaid Services. ICD-10 codes were converted to their category diagnoses defined as all columns to the left of the decimal point. Each patient was considered to have or not have each category diagnosis prior to the NAAT. A computer program calculated the number of cases for each category diagnosis, the relative risk $(R R)$ of death, and its confidence interval (Cl) using a Bonferroni adjustment for multiple comparisons. RRs were re-centered by subtracting 1 so that high-risk conditions had a positive value while protective conditions had a negative one. Diagnoses found to be significant were entered into a logistic model for death in a stepwise fashion. Each patient was assigned (RR-1) to each category diagnosis if they had the condition or 0 otherwise. The resulting model was used to derive PDeathDx for each patient and the area under its receiver operating characteristic $(\mathrm{ROC})$ curve calculated. Single variable logistic models were also derived for age at diagnosis, the Charlson 2-year (Charl2Yr) and lifetime (CharlEver) scores, and the Elixhauser 2-year (Elix2Yrs) and lifetime (ElixEver) scores. Stata was used to compare the ROCs for PDeathDx and each of the other metrics.

Results: On September 30, 2021 there were 347,220 COVID-19 patients in the CSDR. 18,120 patients $(5.33 \%)$ died within 60 days of their diagnosis. After consolidating ICD-9 and ICD-10 
medRxiv preprint doi: https://doi.org/10.1101/2022.01.22.22269694; this version posted February 21, 2022. The copyright holder for this preprint

(which was not certified by peer review) is the author/funder, who has granted medRxiv a license to display the preprint in perpetuity.

This article is a US Government work. It is not subject to copyright under 17 USC 105 and is also made available for use under a CCO license.

COMORBIDITIES IN MODELS FOR COVID-19 DEATH

codes, $29,162,710$ separate diagnoses were given to the subjects representing 41,341 ICD-10 codes. This set was reduced to 1,890 category diagnoses assigned to the group for the first time on $19,184,437$ occasions. Of the 1,890 category diagnoses, 425 involved $>=100$ subjects and had a lower boundary for the $\mathrm{Cl}>=1.50$ (a high-risk condition) or upper boundary $<=0.80$ (a protective condition). Stepwise logistic regression showed that 153 were statistically significant, independent predictors of death. PDeathDx was slightly less powerful than age as a discriminator $(\mathrm{ROC}=0.811+/-0.002$ vs $0.812+/-0.001$, respectively; $\mathrm{P}<0.001)$ but was superior to the Charl2Yr $(\mathrm{ROC}=0.727+/-0.002$; $\mathrm{P}<0.001)$, CharlEver $(\mathrm{ROC}=0.753+/-$ 0.002; $\mathrm{P}<=0.001)$, Elix2Yr $(\mathrm{ROC}=0.694+/-0.002 ; \mathrm{P}<0.001)$; and ElixEver $(\mathrm{ROC}=0.731+/-$ $0.002 ; \mathrm{P}<0.001)$. Univariate analysis and multivariate modeling showed that many of the most high-risk conditions are under-represented or not included in the Charlson Index. These include hypertension, dementia, degenerative neurologic disease, or diagnoses associated with severe physical disability.

Conclusions: Our method for handling pre-existing conditions in multivariate analysis has many advantages over conventional comorbidity indices. The approach can be applied to any condition or outcome, can use any categorical predictors including medications, creates its own condition weights, handles rare as well as protective conditions, and returns actionable information to providers. The latter include the specific ICD-10 groups, their contribution to the risk, and their rank order of importance. Finally, PDeathDx is equivalent to age as a discriminator of outcomes and outperforms 4 other comorbidity scores. If validated by others, this approach provides an alternative and more robust approach to handling comorbidities in multivariate models. 
medRxiv preprint doi: https://doi.org/10.1101/2022.01.22.22269694; this version posted February 21, 2022. The copyright holder for this preprint

(which was not certified by peer review) is the author/funder, who has granted medRxiv a license to display the preprint in perpetuity.

This article is a US Government work. It is not subject to copyright under 17 USC 105 and is also made available for use under a CCO license.

COMORBIDITIES IN MODELS FOR COVID-19 DEATH

\section{INTRODUCTION}

Many mathematical models have been proposed for predicting death from COVID-19 infection (1-5). They have gained popularity because they are very useful for managing patients and allocating scarce resources. One of the most important components of these models is the set of pre-existing conditions. Many diseases increase the mortality rate because they diminish the host response to infection, cause end-organ dysfunction that is further compromised by COVID-19, or severely limit life expectancy and functional status. One method for handling these conditions is to gather them under broad categories ("malignancy") and derive a regression coefficient for each grouping $(3,4)$. Another approach is to use a comorbidity scoring system such as the Charlson Comorbidity Index (CCI) or Elixhauser (ELIX) score (1, 2, 5). With this approach, the groupings are assigned weights, and the weights are added to generate a summary score. The summary score is then used as a covariate in the model. $\mathrm{CCl}$ and ELIX have been extensively validated and applied to many conditions (6). Moreover, mathematical modeling has shown that they provide all that is necessary to handle confounding from the underlying diagnoses (7). However, there are still problems with either approach:

1) $\mathrm{CCl}$ was developed to predict mortality one year after hospitalization. It is less clear that the same conditions are the most influential for outcomes mediated by an immune response such as breakthrough infections.

2) Rare diseases are not well represented. As a result, a person with a common disease of limited impact could be given a poorer prognosis than another with a rare but lethal condition.

3) There is often little evidence that members of each group have the same prognostic significance.

4) Neither method handles conditions that are protective.

5) These approaches do not return actionable information to clinicians. While they may become aware of their patients' risk, providers are not informed of the sources of such 
medRxiv preprint doi: https://doi.org/10.1101/2022.01.22.22269694; this version posted February 21, 2022. The copyright holder for this preprint

(which was not certified by peer review) is the author/funder, who has granted medRxiv a license to display the preprint in perpetuity.

This article is a US Government work. It is not subject to copyright under 17 USC 105 and is also made available for use under a CCO license.

COMORBIDITIES IN MODELS FOR COVID-19 DEATH

risk. As a result, they cannot take steps to mitigate that risk through further studies or treatments.

A more sophisticated approach is to do a systematic survey of all pre-existing conditions, determine which have an impact on outcomes, and generate a predicted probability of death that represents the aggregate risk posed by those that are statistically significant. Like a propensity score, this predicted probability of death (PDeathDx) can be used as a variable in the model. Fortunately, advanced computer methods have made it possible to generate these estimates. The purpose of this study is to describe a novel approach to handling comorbidities in COVID-19 prediction models and compare the performance of PDeathDx with conventional comorbidity measures in distinguishing fatal from non-fatal cases.

\section{METHODS}

Cases were identified through VA's COVID-19 Shared Data Resource (CSDR). Membership in this registry requires at least one positive nucleic acid amplification test (NAAT). The primary outcome was death within 60 days of the diagnosis. The outcome was retrieved from the post-index conditions file of the CSDR, which assigns a 1 to those who died and 0 otherwise. Likewise, the 2-year CCI score (Charl2Yrs), lifetime CCI score (CharlEver), 2-year ELIX score (Elix2Yrs), and lifetime ELIX score (ElixEver) were retrieved from CSDR for each patient. Pre-existing conditions were identified by reviewing all diagnoses entered into the electronic medical record for outpatient visits, on patient problem lists, or at the time of hospital discharge. "Pre-existing" refers to entries made at least 14 days prior to the diagnosis of COVID-19. This precaution excludes any entries that may have been made during the presymptomatic phases of COVID-19. ICD-9 codes were converted to ICD-10 using a crosswalk provided by the Centers for Medicare/Medicaid Services. A "category diagnosis" was defined as all characters preceding the decimal point for ICD-10 codes or the ICD-9 equivalent. A patient was considered to have or not have each category condition prior to the COVID diagnosis. A computer program was used to identify all patients with a given condition who died 
medRxiv preprint doi: https://doi.org/10.1101/2022.01.22.22269694; this version posted February 21, 2022. The copyright holder for this preprint

(which was not certified by peer review) is the author/funder, who has granted medRxiv a license to display the preprint in perpetuity.

This article is a US Government work. It is not subject to copyright under 17 USC 105 and is also made available for use under a CCO license.

COMORBIDITIES IN MODELS FOR COVID-19 DEATH

or survived as well as all patients without the condition who died or survived. The software used these cell frequencies to derive the relative risk of death associated with the condition (RR) and its confidence interval $(\mathrm{Cl})$. $\mathrm{Cl}$ were adjusted for multiple comparisons by the Bonferroni method. A category diagnosis was considered to have a significant effect on the outcome if there were at least 100 cases and if the lower limit for the $\mathrm{Cl}$ was $>=1.5$ or the upper limit was $<=0.80$. Another software program was used to create a diagnostic grid in which each patient was assigned a value for all significant category diagnoses. Recall that a condition with a relative risk of 1 has no effect on the outcome. The scale for relative risk was therefore centered on 1 by subtracting 1 from the relative risk and entering that value for the corresponding category condition if present. Those without the condition were treated as if they had a condition with no prognostic significance and assigned a value of 0 . The diagnostic grid was exported to a statistical program (Stata). Stepwise logistic regression was used to identify those that were independently predictive of death. This procedure adjusted the contribution of each condition for the presence of the others, assigned a predicted probability of death to each patient (PDeathDx), and used PDeathDx to generate an area under a Receiver Operating Characteristic curve (ROC area). The product of coefficient * $(R R-1)$ is the contribution of each condition to the logit function if present. It was therefore used to rank order the importance of the category diagnoses. Differences in categorical variables were tested by chi-square analysis; differences in continuous variables were analyzed by the unpaired student's t-test or rank sum test. Separate logistic models were used to examine the effect of age, Charl2Yrs, CharlEver, Elix2Yrs, and ElixEver as single predictors of death. Stata provides a function that compares the ROC areas for pairs of these variables.

\section{RESULTS}

On September 30, 2021, there were 347,220 COVID-19 patients in VA's COVID-19 Shared Data Resource. 339,772 (or $97.9 \%$ ) had at least one pre-existing condition and form the basis for this report. The mean age at the time of diagnosis was $58.6 \pm 16.7$ years; $84.1 \%$ 
medRxiv preprint doi: https://doi.org/10.1101/2022.01.22.22269694; this version posted February 21, 2022. The copyright holder for this preprint

(which was not certified by peer review) is the author/funder, who has granted medRxiv a license to display the preprint in perpetuity.

This article is a US Government work. It is not subject to copyright under 17 USC 105 and is also made available for use under a CCO license.

COMORBIDITIES IN MODELS FOR COVID-19 DEATH

were male; $22.9 \%$ were members of a racial minority; $9.0 \%$ were Hispanic; $95.8 \%$ were veterans; $0.7 \%$ were on supplemental oxygen; and $11.8 \%$ were current smokers. $9.1 \%$ had been fully vaccinated at least 14 days prior to the COVID-19 diagnosis. $21.5 \%$ acquired their infections after July 1, 2021 and were presumed to have the delta variant. Overall, 18,120 patients $(5.33 \%)$ died within 60 days of their diagnosis.

For the study cohort, 82,578,233 ICD-9 codes had been entered into the electronic record before the VA converted to ICD-10 codes in 2015 . Of these, $81,671,483$ (or $98.9 \%$ ) were successfully converted to an ICD-10 equivalent using the CMS crosswalk. In addition, $78,976,269$ ICD-10 codes were entered at least 14 days prior to the COVID-19 diagnosis. After consolidation, $29,162,710$ separate diagnoses were given to the subjects representing 41,341 ICD-10 codes. The sample size was insufficient to test each ICD-10 code for its prognostic significance. The individual codes were therefore reduced to 1,890 category diagnoses which were assigned to the group for the first time on $19,184,437$ occasions.

Of the 1,890 category diagnoses, 425 involved $\geq 100$ subjects and had a lower boundary for the $\mathrm{Cl}>=1.50$ or upper boundary $<=0.80$ (Table 1$)$. One diagnosis (Z11: screening for other viral diseases) was given to 120,308 subjects and had a high RR for death; it was removed because it may have been used for COVID-19 testing before there was a suitable ICD10 code. Preliminary regressions indicated that another 26 provided a perfect prediction of the outcome when present, while 20 were affected by collinearity. A diagnostic grid was therefore assembled where each patient was assigned a value for each of the remaining 378 category diagnoses. If the diagnosis was present, $(R R-1)$ was assigned as its value and 0 otherwise. Stepwise logistic regression showed that 153 were statistically significant, independent predictors of death (Table 2). PDeathDx was derived for each patient and its ROC area determined. This analysis showed that PDeathDx provided excellent discrimination between those who died and those who survived $(\mathrm{ROC}$ area $=0.811 \pm 0.002)$. Single variable logistic models were also constructed for age at diagnosis, Charl2Yrs, CharlEver, Elix2Yrs, and 
medRxiv preprint doi: https://doi.org/10.1101/2022.01.22.22269694; this version posted February 21, 2022. The copyright holder for this preprint

(which was not certified by peer review) is the author/funder, who has granted medRxiv a license to display the preprint in perpetuity.

This article is a US Government work. It is not subject to copyright under 17 USC 105 and is also made available for use under a CCO license.

COMORBIDITIES IN MODELS FOR COVID-19 DEATH

ElixEver, and ROC areas determined for their predicted probabilities. PDeathDx was less powerful than age as a discriminator $(R O C=0.812 \pm 0.001 ; P<0.001)$ but was superior to the Charl2Yr $(R O C=0.727 \pm 0.002 ; P<0.001)$, CharlEver $(R O C=0.753 \pm 0.002 ; P<=0.001)$, Elix2Yr $(\mathrm{ROC}=0.694 \pm 0.002 ; \mathrm{P}<0.001)$; and ElixEver $(\mathrm{ROC}=0.731 \pm 0.002 ; \mathrm{P}<0.001)$

Tables 1 and 2 show that this approach provides much more clinical information than the indices. Table 1 ranks the category diagnoses by their $\mathrm{RR}$ on univariate analysis.

Degenerative neurologic disease and severe functional disability are prominently represented within the top 20 most influential conditions. This observation is significant because they are not given much weight in comorbidity indices. On the other hand, only one solid tumor (carcinoma of the ampulla of Vater) appears within the top 20. On the other end of the scale, several functional diagnoses, gynecologic disorders, and sexually transmitted diseases reduced the risk of death. Table 2 shows the ICD-10 codes that were independently predictive of death rank ordered by their contribution to the logit when present. Many high-risk conditions became protective when adjusted for the effects of others. Hypertension was the most important independent risk factor for death and represented a greater threat than coronary artery disease. Degenerative neurologic diseases were prominently represented at the top of the list, while malignancies comprised the bulk of high-risk conditions.

\section{DISCUSSION}

We propose a novel method for handling pre-existing conditions in COVID prediction models that has several theoretical advantages over conventional comorbidity indices. Table 3 compares the attributes of the standard comorbidity indices and PDeathDx. Unlike a standard index, our approach creates a different model for every disease. The outcome can be changed by re-defining a target group. Univariate statistical analysis is used to assign condition weights. The method can be applied to any type of categorical predictor including medications and procedures. It handles rare as well as protective conditions. In addition to identifying high-risk codes, the output includes a probability for each pattern of conditions. It can therefore detect 
medRxiv preprint doi: https://doi.org/10.1101/2022.01.22.22269694; this version posted February 21, 2022. The copyright holder for this preprint

(which was not certified by peer review) is the author/funder, who has granted medRxiv a license to display the preprint in perpetuity.

This article is a US Government work. It is not subject to copyright under 17 USC 105 and is also made available for use under a CCO license.

COMORBIDITIES IN MODELS FOR COVID-19 DEATH

circumstances where disease combinations are problematic. Finally, the model can be used to return actional information to providers. Instead of total or condition scores, this information includes the predicted probability of the outcome, the specific diagnoses contributing to the prediction, and their rank order of importance. Such information may prompt the provider to explore a mechanism of injury or an intervention to mitigate the risk.

However, PDeathDx requires programming at a high level, takes more time, and uses more computing resources. The first hurdle is the systematic search for candidate diagnoses among millions of entries in the electronic record and performing screening statistical tests on thousands of root diagnoses. The second is creating a diagnostic array in which each patient is given a score for every significant diagnosis experienced by the cohort. The table must then be transferred to a statistical program capable of handling hundreds of predictors and large number of rows.

PDeathDx provided powerful discrimination between COVID-19 patients who died and survived, out-performed the other comorbidity indices, had an ROC area equivalent to that generated by age, and remains the second most powerful predictor of all those that we have examined thus far. The most influential category conditions included entries not usually included in comorbidity indices. This observation suggests that it is risky to pick comorbidities for analysis without a systematic review of all those experienced by the cohort. Moreover, poor prognoses were assigned to those with dementia, degenerative neurological diseases, and severe disabilities - conditions not usually associated with cardiorespiratory injury or impaired immunity. These disorders are often associated with a poor quality of life that dictates a conservative approach to treatment. If this decision is common for COVID-19 patients, then death may be more indicative of the patient's baseline condition than severity of illness. In that event, this outcome may not be suitable for studies of interventions. Finally, multivariate analysis showed that some high-risk conditions became protective when adjusted for the effect 
medRxiv preprint doi: https://doi.org/10.1101/2022.01.22.22269694; this version posted February 21, 2022. The copyright holder for this preprint

(which was not certified by peer review) is the author/funder, who has granted medRxiv a license to display the preprint in perpetuity.

This article is a US Government work. It is not subject to copyright under 17 USC 105 and is also made available for use under a CCO license.

COMORBIDITIES IN MODELS FOR COVID-19 DEATH

of other components of the model. It would have been a mistake to treat the effect of these conditions as independent and additive.

We did not include age in our model for PDeathDx for 3 reasons. First, it was intended to represent pre-existing conditions in an overarching model containing multiple domains. One domain was comprised of demographic characteristics including age at diagnosis. Next, we did not want age to displace the diagnoses highly correlated with age in the model. To understand the mechanisms that lead to a fatal outcome, explanatory variables should take precedence over disease markers. If age is modelled at all, it should be for a residual effect once causal factors have been included. Finally, age-based models do not support decision-making at the point of care. For example, a physician might feel comfortable withdrawing care if the patient had Alzheimer's disease - but not simply because the patient was old. Clinicians make recommendations based upon an assessment of the underlying conditions. It is the patient's prerogative to decide what is appropriate based upon age and quality of life.

We included all diagnoses in the medical record because some conditions have an effect over the patient's lifetime even if they have not been recently active. Examples include intravenous drug use or sickle cell trait. We also wanted to test our computing resources to see if they were capable of handling problems of this size. Finally, to develop the most efficient model, it is reasonable to start with a comprehensive list of diagnoses and work backwards. That strategy allows one to determine if time-limited sampling compromises the ability of PDeathDx to discriminate between survivors and non-survivors. On the other hand, starting with a fixed time frame for diagnoses is arbitrary and provides no information about the benefits of more remote data. Fortunately, our computing resources were more than sufficient to handle all pre-existing conditions.

The major limitation of our approach is that it handles all pre-existing diagnoses - not just the most recent ones. Thus, a person with chronic renal failure (CRF) who undergoes a transplant and regains normal renal function will still be included in the analysis of CRF. Of 
medRxiv preprint doi: https://doi.org/10.1101/2022.01.22.22269694; this version posted February 21, 2022. The copyright holder for this preprint

(which was not certified by peer review) is the author/funder, who has granted medRxiv a license to display the preprint in perpetuity.

This article is a US Government work. It is not subject to copyright under 17 USC 105 and is also made available

COMORBIDITIES IN MODELS FOR COVID-19 DEATH

course, our conclusions are limited to patients with characteristics like the veteran population.

Further studies should be done on other populations and disease states before the method

should be widely applied. If validated by others, our method could provide a more robust

alternative to comorbidity scores for handling pre-existing conditions in multivariate models. 
medRxiv preprint doi: https://doi.org/10.1101/2022.01.22.22269694; this version posted February 21, 2022. The copyright holder for this preprint (which was not certified by peer review) is the author/funder, who has granted medRxiv a license to display the preprint in perpetuity. This article is a US Government work. It is not subject to copyright under 17 USC 105 and is also made available for use under a CCO license.

\section{TABLE 1: ICD-10 CATEGORY DIAGNOSES PREDICTIVE OF DEATH ON UNIVARIATE ANALYSIS}

\section{${ }^{*}$ Most Common ICD-10 Code and Description for Each Category Diagnosis}

\begin{tabular}{|c|c|c|c|c|c|c|c|c|}
\hline $\begin{array}{c}\text { Root } \\
\text { ICD-10 }\end{array}$ & $\begin{array}{l}\text { ICD-10 } \\
\text { Code }^{*}\end{array}$ & ICD-10 Description* & $\begin{array}{l}\text { No. of } \\
\text { Cases }\end{array}$ & $\begin{array}{l}\text { Case } \\
\text { Rate }\end{array}$ & $\begin{array}{c}\text { Control } \\
\text { Rate }\end{array}$ & $\begin{array}{l}\text { Relative } \\
\text { Risk }\end{array}$ & $\begin{array}{l}\text { Lower } \\
\text { Cl }\end{array}$ & $\begin{array}{l}\text { Higher } \\
\text { Cl }\end{array}$ \\
\hline G30 & G30.9 & Alzheimer's disease, unspecified & 4348 & 0.2672 & 0.0506 & 5.2861 & 4.7347 & 5.9017 \\
\hline F03 & F03.90 & $\begin{array}{l}\text { Unspecified dementia without } \\
\text { behavioral disturbance }\end{array}$ & 13895 & 0.2340 & 0.0456 & 5.1278 & 4.7678 & 5.5150 \\
\hline F02 & F02.80 & $\begin{array}{l}\text { Dementia in oth diseases classd } \\
\text { elswhr w/o behavrl disturb }\end{array}$ & 8684 & 0.2379 & 0.0485 & 4.9065 & 4.4977 & 5.3524 \\
\hline 110 & 110. & Essential (primary) hypertension & 208817 & 0.0768 & 0.0159 & 4.8387 & 4.3920 & 5.3309 \\
\hline F01 & F01.50 & $\begin{array}{l}\text { Vascular dementia without } \\
\text { behavioral disturbance }\end{array}$ & 6033 & 0.2342 & 0.0501 & 4.6786 & 4.2212 & 5.1856 \\
\hline Z66 & Z66. & Do not resuscitate & 6448 & 0.2244 & 0.0500 & 4.4864 & 4.0498 & 4.9700 \\
\hline $\mathrm{R} 62$ & R62.7 & Adult failure to thrive & 3414 & 0.2209 & 0.0516 & 4.2777 & 3.7238 & 4.9140 \\
\hline R54 & R54. & Age-related physical debility & 11357 & 0.1983 & 0.0483 & 4.1040 & 3.7668 & 4.4714 \\
\hline C93 & C93.10 & $\begin{array}{l}\text { Chronic myelomonocytic } \\
\text { leukemia not achieve remission }\end{array}$ & 122 & 0.2131 & 0.0533 & 4.0005 & 1.9240 & 8.3180 \\
\hline D46 & D46.9 & $\begin{array}{l}\text { Myelodysplastic syndrome, } \\
\text { unspecified }\end{array}$ & 755 & 0.2106 & 0.0530 & 3.9750 & 2.9513 & 5.3539 \\
\hline L89 & L89.90 & $\begin{array}{l}\text { Pressure ulcer of unspecified } \\
\text { site, unspecified stage }\end{array}$ & 8092 & 0.1908 & 0.0500 & 3.8180 & 3.4499 & 4.2253 \\
\hline 113 & 113.0 & $\begin{array}{l}\text { Hyp hrt \& chr kdny dis w hrt fail } \\
\text { and stg 1-4/unsp chr kdny }\end{array}$ & 9823 & 0.1844 & 0.0494 & 3.7299 & 3.3925 & 4.1008 \\
\hline J64 & J64. & Unspecified pneumoconiosis & 107 & 0.1963 & 0.0533 & 3.6833 & 1.6173 & 8.3882 \\
\hline C95 & C95.90 & $\begin{array}{l}\text { Leukemia, unspecified not } \\
\text { having achieved remission }\end{array}$ & 609 & 0.1938 & 0.0531 & 3.6505 & 2.5754 & 5.1743 \\
\hline R64 & R64. & Cachexia & 797 & 0.1932 & 0.0530 & 3.6457 & 2.6851 & 4.9499 \\
\hline E43 & E43. & $\begin{array}{l}\text { Unspecified severe protein- } \\
\text { calorie malnutrition }\end{array}$ & 1597 & 0.1904 & 0.0527 & 3.6133 & 2.9023 & 4.4984 \\
\hline $\mathrm{Z74}$ & Z74.09 & Other reduced mobility & 17940 & 0.1681 & 0.0469 & 3.5822 & 3.3154 & 3.8704 \\
\hline D63 & D63.1 & $\begin{array}{l}\text { Anemia in chronic kidney } \\
\text { disease }\end{array}$ & 14823 & 0.1703 & 0.0480 & 3.5494 & 3.2667 & 3.8565 \\
\hline C24 & $\mathrm{C} 24.1$ & $\begin{array}{l}\text { Malignant neoplasm of ampulla } \\
\text { of Vater }\end{array}$ & 122 & 0.1885 & 0.0533 & 3.5383 & 1.6054 & 7.7983 \\
\hline F05 & F05. & $\begin{array}{l}\text { Delirium due to known } \\
\text { physiological condition }\end{array}$ & 3612 & 0.1822 & 0.0519 & 3.5070 & 3.0142 & 4.0803 \\
\hline Y95 & Y95. & Nosocomial condition & 355 & 0.1859 & 0.0532 & 3.4952 & 2.1891 & 5.5806 \\
\hline W06 & $\begin{array}{l}\text { W06.XX } \\
\text { XA }\end{array}$ & Fall from bed, initial encounter & 2167 & 0.1832 & 0.0525 & 3.4898 & 2.8768 & 4.2334 \\
\hline C78 & C78.7 & $\begin{array}{l}\text { Secondary malig neoplasm of } \\
\text { liver and intrahepatic bile duct }\end{array}$ & 1305 & 0.1839 & 0.0528 & 3.4814 & 2.7193 & 4.4570 \\
\hline J90 & J90. & $\begin{array}{l}\text { Pleural effusion, not elsewhere } \\
\text { classified }\end{array}$ & 5673 & 0.1754 & 0.0513 & 3.4218 & 3.0197 & 3.8774 \\
\hline J69 & J69.0 & $\begin{array}{l}\text { Pneumonitis due to inhalation of } \\
\text { food and vomit }\end{array}$ & 2896 & 0.1785 & 0.0523 & 3.4165 & 2.8812 & 4.0511 \\
\hline 150 & 150.9 & Heart failure, unspecified & 36427 & 0.1447 & 0.0424 & 3.4162 & 3.2021 & 3.6445 \\
\hline N18 & N18.9 & $\begin{array}{l}\text { Chronic kidney disease, } \\
\text { unspecified }\end{array}$ & 48428 & 0.1352 & 0.0397 & 3.4042 & 3.2006 & 3.6206 \\
\hline 175 & 175.029 & $\begin{array}{l}\text { Atheroembolism of unspecified } \\
\text { lower extremity }\end{array}$ & 138 & 0.1812 & 0.0533 & 3.4003 & 1.5879 & 7.2814 \\
\hline J81 & J81.0 & Acute pulmonary edema & 2462 & 0.1767 & 0.0524 & 3.3700 & 2.7995 & 4.0567 \\
\hline G20 & G20. & Parkinson's disease & 5105 & 0.1732 & 0.0515 & 3.3623 & 2.9456 & 3.8379 \\
\hline N25 & N25.81 & $\begin{array}{l}\text { Secondary hyperparathyroidism } \\
\text { of renal origin }\end{array}$ & 6475 & 0.1699 & 0.0511 & 3.3268 & 2.9515 & 3.7497 \\
\hline J96 & J96.01 & $\begin{array}{l}\text { Acute respiratory failure with } \\
\text { hypoxia }\end{array}$ & 13350 & 0.1624 & 0.0489 & 3.3231 & 3.0408 & 3.6316 \\
\hline
\end{tabular}


medRxiv preprint doi: https://doi.org/10.1101/2022.01.22.22269694; this version posted February 21, 2022. The copyright holder for this preprint (which was not certified by peer review) is the author/funder, who has granted medRxiv a license to display the preprint in perpetuity. This article is a US Government work. It is not subject to copyright under 17 USC 105 and is also made available for use under a CCO license.

\begin{tabular}{|c|c|c|c|c|c|c|c|c|}
\hline 112 & I12.9 & $\begin{array}{l}\text { Hypertensive chronic kidney } \\
\text { disease w stg } 1-4 / \text { unsp chr kdny }\end{array}$ & 26422 & 0.1498 & 0.0452 & 3.3156 & 3.0895 & 3.5582 \\
\hline D02 & D02.20 & $\begin{array}{l}\text { Carcinoma in situ of unspecified } \\
\text { bronchus and lung }\end{array}$ & 2023 & 0.1740 & 0.0526 & 3.3075 & 2.6919 & 4.0639 \\
\hline W07 & $\begin{array}{l}\text { W07.XX } \\
\text { XA }\end{array}$ & Fall from chair, initial encounter & 1948 & 0.1740 & 0.0526 & 3.3063 & 2.6807 & 4.0780 \\
\hline C94 & C94.6 & $\begin{array}{l}\text { Myelodysplastic disease, not } \\
\text { classified }\end{array}$ & 412 & 0.1748 & 0.0532 & 3.2860 & 2.0929 & 5.1592 \\
\hline N19 & N19. & Unspecified kidney failure & 7383 & 0.1663 & 0.0508 & 3.2729 & 2.9203 & 3.6680 \\
\hline L12 & L12.0 & Bullous pemphigoid & 334 & 0.1737 & 0.0532 & 3.2634 & 1.9740 & 5.3952 \\
\hline R26 & R26.9 & $\begin{array}{l}\text { Unspecified abnormalities of gait } \\
\text { and mobility }\end{array}$ & 67067 & 0.1202 & 0.0369 & 3.2578 & 3.0675 & 3.4598 \\
\hline Z49 & Z49.31 & $\begin{array}{l}\text { Encounter for adequacy testing } \\
\text { for hemodialysis }\end{array}$ & 1540 & 0.1714 & 0.0528 & 3.2472 & 2.5607 & 4.1178 \\
\hline 196 & 196. & $\begin{array}{l}\text { Gangrene, not elsewhere } \\
\text { classified }\end{array}$ & 2079 & 0.1708 & 0.0526 & 3.2459 & 2.6429 & 3.9864 \\
\hline $\mathrm{H} 27$ & H27.8 & Other specified disorders of lens & 6825 & 0.1642 & 0.0511 & 3.2170 & 2.8561 & 3.6236 \\
\hline 125 & I25.10 & $\begin{array}{l}\text { Athscl heart disease of native } \\
\text { coronary artery w/o ang pctrs }\end{array}$ & 77259 & 0.1139 & 0.0355 & 3.2083 & 3.0212 & 3.4069 \\
\hline T66 & $\begin{array}{c}\text { T66.XXX } \\
\text { A }\end{array}$ & $\begin{array}{l}\text { Radiation sickness, unspecified, } \\
\text { initial encounter }\end{array}$ & 211 & 0.1706 & 0.0533 & 3.2036 & 1.6912 & 6.0686 \\
\hline C34 & C34.90 & $\begin{array}{l}\text { Malignant neoplasm of unsp part } \\
\text { of unsp bronchus or lung }\end{array}$ & 3805 & 0.1664 & 0.0520 & 3.1962 & 2.7354 & 3.7346 \\
\hline E46 & E46. & $\begin{array}{l}\text { Unspecified protein-calorie } \\
\text { malnutrition }\end{array}$ & 3568 & 0.1659 & 0.0521 & 3.1825 & 2.7098 & 3.7377 \\
\hline E44 & E44.0 & $\begin{array}{l}\text { Moderate protein-calorie } \\
\text { malnutrition }\end{array}$ & 3752 & 0.1647 & 0.0521 & 3.1623 & 2.7011 & 3.7022 \\
\hline C91 & C91.10 & $\begin{array}{l}\text { Chronic lymphocytic leuk of B- } \\
\text { cell type not achieve remis }\end{array}$ & 1854 & 0.1661 & 0.0527 & 3.1517 & 2.5270 & 3.9307 \\
\hline N17 & N17.9 & Acute kidney failure, unspecified & 31785 & 0.1396 & 0.0444 & 3.1421 & 2.9347 & 3.3641 \\
\hline Z19 & Z19.2 & $\begin{array}{l}\text { Hormone resistant malignancy } \\
\text { status }\end{array}$ & 174 & 0.1667 & 0.0533 & 3.1286 & 1.5331 & 6.3847 \\
\hline L22 & L22. & Diaper dermatitis & 774 & 0.1654 & 0.0531 & 3.1159 & 2.2159 & 4.3814 \\
\hline$\overline{\text { G91 }}$ & G91.2 & $\begin{array}{l}\text { (Idiopathic) normal pressure } \\
\text { hydrocephalus }\end{array}$ & 917 & 0.1647 & 0.0530 & 3.1053 & 2.2680 & 4.2515 \\
\hline G92 & G92. & Toxic encephalopathy & 1085 & 0.1631 & 0.0530 & 3.0793 & 2.3025 & 4.1180 \\
\hline Y73 & Y73.2 & $\begin{array}{l}\text { Prosth/oth implnt/mtrls gastroent } \\
\text { and urol dev assoc w incdt }\end{array}$ & 735 & 0.1633 & 0.0531 & 3.0752 & 2.1619 & 4.3743 \\
\hline C79 & C79.51 & $\begin{array}{l}\text { Secondary malignant neoplasm } \\
\text { of bone }\end{array}$ & 2724 & 0.1612 & 0.0525 & 3.0721 & 2.5499 & 3.7014 \\
\hline Z96 & $Z 96.1$ & Presence of intraocular lens & 58557 & 0.1206 & 0.0393 & 3.0656 & 2.8838 & 3.2588 \\
\hline E78 & E78.5 & Hyperlipidemia, unspecified & 223873 & 0.0692 & 0.0226 & 3.0600 & 2.8037 & 3.3397 \\
\hline 173 & 173.9 & $\begin{array}{l}\text { Peripheral vascular disease, } \\
\text { unspecified }\end{array}$ & 31350 & 0.1369 & 0.0448 & 3.0545 & 2.8506 & 3.2730 \\
\hline $\mathrm{R} 18$ & R18.8 & Other ascites & 2408 & 0.1586 & 0.0526 & 3.0172 & 2.4710 & 3.6840 \\
\hline C66 & C66.9 & $\begin{array}{l}\text { Malignant neoplasm of } \\
\text { unspecified ureter }\end{array}$ & 237 & 0.1603 & 0.0533 & 3.0107 & 1.6104 & 5.6286 \\
\hline Z95 & Z95.1 & $\begin{array}{l}\text { Presence of aortocoronary } \\
\text { bypass graft }\end{array}$ & 34407 & 0.1330 & 0.0444 & 2.9986 & 2.8020 & 3.2089 \\
\hline $\mathrm{Z71}$ & Z71.89 & Other specified counseling & 308757 & 0.0568 & 0.0190 & 2.9898 & 2.5115 & 3.5592 \\
\hline J91 & J91.8 & $\begin{array}{l}\text { Pleural effusion in other } \\
\text { conditions classified elsewhere }\end{array}$ & 4873 & 0.1545 & 0.0519 & 2.9798 & 2.5795 & 3.4422 \\
\hline G11 & G11.1 & Early-onset cerebellar ataxia & 586 & 0.1570 & 0.0532 & 2.9538 & 1.9729 & 4.4224 \\
\hline 148 & 148.91 & Unspecified atrial fibrillation & 35951 & 0.1301 & 0.0442 & 2.9419 & 2.7502 & 3.1470 \\
\hline $\mathrm{J} 15$ & $J 15.9$ & Unspecified bacterial pneumonia & 9858 & 0.1482 & 0.0505 & 2.9350 & 2.6389 & 3.2644 \\
\hline N14 & $\mathrm{N} 14.0$ & Analgesic nephropathy & 405 & 0.1556 & 0.0532 & 2.9235 & 1.7952 & 4.7611 \\
\hline 167 & 167.89 & Other cerebrovascular disease & 16692 & 0.1420 & 0.0487 & 2.9125 & 2.6714 & 3.1754 \\
\hline $\mathrm{H} 26$ & $\mathrm{H} 26.9$ & Unspecified cataract & 60786 & 0.1156 & 0.0398 & 2.9053 & 2.7326 & 3.0889 \\
\hline
\end{tabular}


medRxiv preprint doi: https://doi.org/10.1101/2022.01.22.22269694; this version posted February 21, 2022. The copyright holder for this preprint (which was not certified by peer review) is the author/funder, who has granted medRxiv a license to display the preprint in perpetuity. This article is a US Government work. It is not subject to copyright under 17 USC 105 and is also made available for use under a CCO license.

\begin{tabular}{|c|c|c|c|c|c|c|c|c|}
\hline $\begin{array}{l}71 \\
\text { Y71 }\end{array}$ & Y71.2 & $\begin{array}{l}\text { Prosth/oth implnt/mtrls } \\
\text { cardiovascular devices assoc w } \\
\text { incdt }\end{array}$ & 989 & 0.1537 & 0.0530 & 2.8978 & 2.1144 & 3.9715 \\
\hline J17 & J17. & $\begin{array}{l}\text { Pneumonia in diseases classified } \\
\text { elsewhere }\end{array}$ & 2185 & 0.1524 & 0.0527 & 2.8925 & 2.3345 & 3.5838 \\
\hline J61 & J61. & $\begin{array}{l}\text { Pneumoconiosis due to asbestos } \\
\text { and other mineral fibers }\end{array}$ & 1189 & 0.1531 & 0.0530 & 2.8892 & 2.1653 & 3.8551 \\
\hline T82 & $\begin{array}{c}\text { T82.818 } \\
\text { A }\end{array}$ & $\begin{array}{l}\text { Embolism due to vascular prosth } \\
\text { dev/grft, initial encounter }\end{array}$ & 6593 & 0.1479 & 0.0515 & 2.8738 & 2.5282 & 3.2668 \\
\hline 195 & 195.9 & Hypotension, unspecified & 32131 & 0.1301 & 0.0453 & 2.8710 & 2.6771 & 3.0789 \\
\hline 170 & 170.0 & Atherosclerosis of aorta & 19489 & 0.1379 & 0.0482 & 2.8613 & 2.6354 & 3.1065 \\
\hline 127 & 127.20 & $\begin{array}{l}\text { Pulmonary hypertension, } \\
\text { unspecified }\end{array}$ & 9999 & 0.1445 & 0.0506 & 2.8580 & 2.5677 & 3.1811 \\
\hline W90 & $\begin{array}{l}\text { W90.8XX } \\
\text { A }\end{array}$ & $\begin{array}{l}\text { Exposure to other nonionizing } \\
\text { radiation, initial encounter }\end{array}$ & 250 & 0.1520 & 0.0533 & 2.8541 & 1.5219 & 5.3522 \\
\hline 162 & 162.00 & $\begin{array}{l}\text { Nontraumatic subdural } \\
\text { hemorrhage, unspecified }\end{array}$ & 1571 & 0.1509 & 0.0529 & 2.8530 & 2.2142 & 3.6762 \\
\hline N40 & N40.0 & $\begin{array}{l}\text { Benign prostatic hyperplasia } \\
\text { without lower urinry tract symp }\end{array}$ & 85767 & 0.1036 & 0.0363 & 2.8518 & 2.6852 & 3.0289 \\
\hline R65 & R65.20 & $\begin{array}{l}\text { Severe sepsis without septic } \\
\text { shock }\end{array}$ & 5450 & 0.1468 & 0.0518 & 2.8334 & 2.4613 & 3.2617 \\
\hline A41 & A41.9 & Sepsis, unspecified organism & 12759 & 0.1412 & 0.0499 & 2.8303 & 2.5681 & 3.1193 \\
\hline $\mathrm{C} 83$ & C83.30 & $\begin{array}{l}\text { Diffuse large B-cell lymphoma, } \\
\text { unspecified site }\end{array}$ & 1195 & 0.1498 & 0.0530 & 2.8268 & 2.1123 & 3.7829 \\
\hline L97 & L97.509 & $\begin{array}{l}\text { Non-pressure chronic ulcer oth } \\
\text { prt unsp foot w unsp severity }\end{array}$ & 13950 & 0.1401 & 0.0496 & 2.8231 & 2.5701 & 3.1010 \\
\hline 106 & 106.0 & Rheumatic aortic stenosis & 614 & 0.1498 & 0.0532 & 2.8189 & 1.8796 & 4.2275 \\
\hline C92 & C92.10 & $\begin{array}{l}\text { Chronic myeloid leuk, BCR/ABL- } \\
\text { positive, not achieve remis }\end{array}$ & 602 & 0.1495 & 0.0532 & 2.8123 & 1.8667 & 4.2369 \\
\hline G31 & G31.84 & $\begin{array}{l}\text { Mild cognitive impairment, so } \\
\text { stated }\end{array}$ & 21486 & 0.1343 & 0.0479 & 2.8064 & 2.5905 & 3.0402 \\
\hline G70 & G70.00 & $\begin{array}{l}\text { Myasthenia gravis without } \\
\text { (acute) exacerbation }\end{array}$ & 745 & 0.1490 & 0.0531 & 2.8049 & 1.9386 & 4.0581 \\
\hline G21 & G21.11 & $\begin{array}{l}\text { Neuroleptic induced } \\
\text { parkinsonism }\end{array}$ & 2043 & 0.1478 & 0.0528 & 2.8019 & 2.2364 & 3.5103 \\
\hline D61 & D61.818 & Other pancytopenia & 3153 & 0.1468 & 0.0525 & 2.7995 & 2.3311 & 3.3619 \\
\hline J70 & J70.5 & $\begin{array}{l}\text { Respiratory conditions due to } \\
\text { smoke inhalation }\end{array}$ & 438 & 0.1484 & 0.0532 & 2.7891 & 1.7221 & 4.5172 \\
\hline R57 & R57.0 & Cardiogenic shock & 1089 & 0.1478 & 0.0530 & 2.7881 & 2.0503 & 3.7914 \\
\hline 163 & 163.9 & Cerebral infarction, unspecified & 18075 & 0.1357 & 0.0487 & 2.7853 & 2.5572 & 3.0338 \\
\hline C19 & C19. & $\begin{array}{l}\text { Malignant neoplasm of } \\
\text { rectosigmoid junction }\end{array}$ & 547 & 0.1481 & 0.0532 & 2.7847 & 1.8074 & 4.2903 \\
\hline H25 & $\mathrm{H} 25.13$ & $\begin{array}{l}\text { Age-related nuclear cataract, } \\
\text { bilateral }\end{array}$ & 127537 & 0.0888 & 0.0320 & 2.7742 & 2.6054 & 2.9539 \\
\hline W08 & $\begin{array}{l}\text { W08.XX } \\
\text { XA }\end{array}$ & $\begin{array}{l}\text { Fall from other furniture, initial } \\
\text { encounter }\end{array}$ & 544 & 0.1471 & 0.0532 & 2.7653 & 1.7896 & 4.2730 \\
\hline $\mathrm{Z75}$ & Z75.1 & $\begin{array}{l}\text { Person awaiting admission to } \\
\text { adequate facility elsewhere }\end{array}$ & 10861 & 0.1394 & 0.0505 & 2.7610 & 2.4854 & 3.0672 \\
\hline S88 & $\begin{array}{c}\text { S88.119 } \\
\text { A }\end{array}$ & $\begin{array}{l}\text { Complete traum amp at lev betw } \\
\text { kn \& ankl, unsp low leg, init }\end{array}$ & 1412 & 0.1459 & 0.0529 & 2.7556 & 2.0985 & 3.6185 \\
\hline J84 & J84.10 & Pulmonary fibrosis, unspecified & 7398 & 0.1414 & 0.0514 & 2.7524 & 2.4303 & 3.1172 \\
\hline N08 & N08. & $\begin{array}{l}\text { Glomerular disorders in diseases } \\
\text { classified elsewhere }\end{array}$ & 2453 & 0.1443 & 0.0527 & 2.7400 & 2.2233 & 3.3768 \\
\hline 165 & 165.29 & $\begin{array}{l}\text { Occlusion and stenosis of } \\
\text { unspecified carotid artery }\end{array}$ & 16848 & 0.1344 & 0.0491 & 2.7367 & 2.5052 & 2.9897 \\
\hline S78 & $\begin{array}{c}\text { S78.019 } \\
\text { A }\end{array}$ & $\begin{array}{l}\text { Complete traumatic amputation } \\
\text { at unsp hip joint, init encntr }\end{array}$ & 447 & 0.1454 & 0.0532 & 2.7329 & 1.6860 & 4.4299 \\
\hline $\mathrm{J} 92$ & J92.9 & Pleural plaque without asbestos & 1007 & 0.1450 & 0.0531 & 2.7326 & 1.9780 & 3.7751 \\
\hline 169 & 169.898 & $\begin{array}{l}\text { Other sequelae of other } \\
\text { cerebrovascular disease }\end{array}$ & 14535 & 0.1356 & 0.0497 & 2.7310 & 2.4866 & 2.9995 \\
\hline
\end{tabular}


medRxiv preprint doi: https://doi.org/10.1101/2022.01.22.22269694; this version posted February 21, 2022. The copyright holder for this preprint (which was not certified by peer review) is the author/funder, who has granted medRxiv a license to display the preprint in perpetuity. This article is a US Government work. It is not subject to copyright under 17 USC 105 and is also made available for use under a CCO license.

\begin{tabular}{|c|c|c|c|c|c|c|c|c|}
\hline D64 & D64.9 & Anemia, unspecified & 62258 & 0.1103 & 0.0405 & 2.7201 & 2.5576 & 2.8930 \\
\hline S72 & $\begin{array}{c}\text { S72.009 } \\
\text { A }\end{array}$ & $\begin{array}{l}\text { Fracture of unsp part of neck of } \\
\text { unsp femur, init }\end{array}$ & 3337 & 0.1423 & 0.0524 & 2.7140 & 2.2641 & 3.2535 \\
\hline $\mathrm{C} 80$ & C80.1 & $\begin{array}{l}\text { Malignant (primary) neoplasm, } \\
\text { unspecified }\end{array}$ & 1345 & 0.1435 & 0.0530 & 2.7089 & 2.0438 & 3.5904 \\
\hline W05 & $\begin{array}{l}\text { W05.0XX } \\
\text { A }\end{array}$ & $\begin{array}{l}\text { Fall from non-moving wheelchair, } \\
\text { initial encounter }\end{array}$ & 660 & 0.1439 & 0.0532 & 2.7080 & 1.8148 & 4.0408 \\
\hline S98 & $\begin{array}{c}\text { S98.119 } \\
\text { A }\end{array}$ & $\begin{array}{l}\text { Complete traumatic amputation } \\
\text { of unsp great toe, init encntr }\end{array}$ & 1353 & 0.1434 & 0.0530 & 2.7069 & 2.0437 & 3.5853 \\
\hline R33 & R33.9 & Retention of urine, unspecified & 20323 & 0.1307 & 0.0484 & 2.7009 & 2.4861 & 2.9343 \\
\hline C71 & C71.9 & $\begin{array}{l}\text { Malignant neoplasm of brain, } \\
\text { unspecified }\end{array}$ & 557 & 0.1436 & 0.0532 & 2.7007 & 1.7462 & 4.1768 \\
\hline J47 & J47.9 & Bronchiectasis, uncomplicated & 1749 & 0.1424 & 0.0529 & 2.6928 & 2.1001 & 3.4529 \\
\hline D53 & D53.9 & Nutritional anemia, unspecified & 4238 & 0.1406 & 0.0522 & 2.6927 & 2.2886 & 3.1682 \\
\hline J44 & J44.9 & $\begin{array}{l}\text { Chronic obstructive pulmonary } \\
\text { disease, unspecified }\end{array}$ & 63653 & 0.1089 & 0.0405 & 2.6858 & 2.5255 & 2.8563 \\
\hline 179 & 179.8 & $\begin{array}{l}\text { Oth disord of art,arterioles \& } \\
\text { capilare in dis classd elswhr }\end{array}$ & 940 & 0.1426 & 0.0531 & 2.6855 & 1.9159 & 3.7643 \\
\hline M80 & $\begin{array}{l}\mathrm{M} 80.88 \mathrm{X} \\
\mathrm{S}\end{array}$ & $\begin{array}{l}\text { Oth osteopor w current path } \\
\text { fracture, vertebra(e), sequela }\end{array}$ & 1227 & 0.1418 & 0.0530 & 2.6752 & 1.9881 & 3.5997 \\
\hline 144 & 144.0 & $\begin{array}{l}\text { Atrioventricular block, first } \\
\text { degree }\end{array}$ & 14983 & 0.1324 & 0.0497 & 2.6653 & 2.4270 & 2.9270 \\
\hline N26 & N26.1 & Atrophy of kidney (terminal) & 757 & 0.1413 & 0.0531 & 2.6602 & 1.8232 & 3.8816 \\
\hline $\mathrm{J} 80$ & J80. & $\begin{array}{l}\text { Acute respiratory distress } \\
\text { syndrome }\end{array}$ & 1161 & 0.1404 & 0.0530 & 2.6474 & 1.9479 & 3.5981 \\
\hline C90 & C90.00 & $\begin{array}{l}\text { Multiple myeloma not having } \\
\text { achieved remission }\end{array}$ & 1041 & 0.1402 & 0.0531 & 2.6431 & 1.9115 & 3.6547 \\
\hline $\mathrm{C} 22$ & $\mathrm{C} 22.0$ & Liver cell carcinoma & 1150 & 0.1400 & 0.0530 & 2.6397 & 1.9385 & 3.5947 \\
\hline Y82 & Y82.8 & $\begin{array}{l}\text { Other medical devices } \\
\text { associated with adverse } \\
\text { incidents }\end{array}$ & 1007 & 0.1400 & 0.0531 & 2.6383 & 1.8972 & 3.6688 \\
\hline 135 & 135.0 & $\begin{array}{l}\text { Nonrheumatic aortic (valve) } \\
\text { stenosis }\end{array}$ & 12454 & 0.1320 & 0.0503 & 2.6225 & 2.3688 & 2.9033 \\
\hline C32 & C32.9 & $\begin{array}{l}\text { Malignant neoplasm of larynx, } \\
\text { unspecified }\end{array}$ & 930 & 0.1387 & 0.0531 & 2.6125 & 1.8504 & 3.6883 \\
\hline 108 & 108.0 & $\begin{array}{l}\text { Rheumatic disorders of both } \\
\text { mitral and aortic valves }\end{array}$ & 3548 & 0.1370 & 0.0524 & 2.6117 & 2.1819 & 3.1263 \\
\hline E11 & E11.9 & $\begin{array}{l}\text { Type } 2 \text { diabetes mellitus without } \\
\text { complications }\end{array}$ & 114394 & 0.0902 & 0.0346 & 2.6085 & 2.4536 & 2.7731 \\
\hline 122 & 122.2 & $\begin{array}{l}\text { Subsequent non-ST elevation } \\
\text { (NSTEMI) myocardial infarction }\end{array}$ & 897 & 0.1382 & 0.0531 & 2.6031 & 1.8311 & 3.7006 \\
\hline 171 & 171.4 & $\begin{array}{l}\text { Abdominal aortic aneurysm, } \\
\text { without rupture }\end{array}$ & 12513 & 0.1311 & 0.0504 & 2.6027 & 2.3505 & 2.8818 \\
\hline 121 & I21.4 & $\begin{array}{l}\text { Non-ST elevation (NSTEMI) } \\
\text { myocardial infarction }\end{array}$ & 14174 & 0.1297 & 0.0500 & 2.5947 & 2.3550 & 2.8588 \\
\hline N04 & N04.9 & $\begin{array}{l}\text { Nephrotic syndrome with } \\
\text { unspecified morphologic } \\
\text { changes }\end{array}$ & 1098 & 0.1375 & 0.0531 & 2.5920 & 1.8837 & 3.5666 \\
\hline $\mathrm{J} 94$ & J94.8 & Other specified pleural conditions & 726 & 0.1377 & 0.0531 & 2.5916 & 1.7519 & 3.8338 \\
\hline D04 & D04.9 & $\begin{array}{l}\text { Carcinoma in situ of skin, } \\
\text { unspecified }\end{array}$ & 3845 & 0.1355 & 0.0524 & 2.5864 & 2.1733 & 3.0780 \\
\hline E87 & E87.6 & Hypokalemia & 54353 & 0.1097 & 0.0426 & 2.5770 & 2.4185 & 2.7459 \\
\hline $\mathrm{R} 60$ & $\mathrm{R} 60.0$ & Localized edema & 61977 & 0.1065 & 0.0415 & 2.5673 & 2.4126 & 2.7320 \\
\hline T45 & $\begin{array}{c}\mathrm{T} 45.1 \times 5 \\
\mathrm{~A}\end{array}$ & $\begin{array}{l}\text { Adverse effect of antineoplastic } \\
\text { and immunosup drugs, init }\end{array}$ & 2220 & 0.1351 & 0.0528 & 2.5598 & 2.0383 & 3.2146 \\
\hline W01 & $\begin{array}{c}\text { W01.0XX } \\
\text { A }\end{array}$ & $\begin{array}{l}\text { Fall same lev from slip/trip w/o } \\
\text { strike against object, init }\end{array}$ & 9034 & 0.1311 & 0.0512 & 2.5594 & 2.2742 & 2.8805 \\
\hline $\mathrm{C} 85$ & C85.80 & $\begin{array}{l}\text { Oth types of non-Hodgkin } \\
\text { lymphoma, unspecified site }\end{array}$ & 2228 & 0.1351 & 0.0528 & 2.5592 & 2.0386 & 3.2127 \\
\hline
\end{tabular}


medRxiv preprint doi: https://doi.org/10.1101/2022.01.22.22269694; this version posted February 21, 2022. The copyright holder for this preprint (which was not certified by peer review) is the author/funder, who has granted medRxiv a license to display the preprint in perpetuity. This article is a US Government work. It is not subject to copyright under 17 USC 105 and is also made available for use under a CCO license.

\begin{tabular}{|c|c|c|c|c|c|c|c|c|}
\hline H34 & H34.219 & $\begin{array}{l}\text { Partial retinal artery occlusion, } \\
\text { unspecified eye }\end{array}$ & 7070 & 0.1321 & 0.0517 & 2.5575 & 2.2414 & 2.9181 \\
\hline J18 & J18.9 & $\begin{array}{l}\text { Pneumonia, unspecified } \\
\text { organism }\end{array}$ & 32422 & 0.1187 & 0.0464 & 2.5551 & 2.3768 & 2.7467 \\
\hline Z45 & $Z 45.2$ & $\begin{array}{l}\text { Encounter for adjustment and } \\
\text { management of VAD }\end{array}$ & 11371 & 0.1290 & 0.0507 & 2.5442 & 2.2854 & 2.8322 \\
\hline 174 & 174.3 & $\begin{array}{l}\text { Embolism and thrombosis of } \\
\text { arteries of the lower extremities }\end{array}$ & 3254 & 0.1337 & 0.0526 & 2.5438 & 2.1033 & 3.0764 \\
\hline T86 & T86.10 & $\begin{array}{l}\text { Unspecified complication of } \\
\text { kidney transplant }\end{array}$ & 1392 & 0.1343 & 0.0530 & 2.5349 & 1.9012 & 3.3798 \\
\hline E08 & E08.42 & $\begin{array}{l}\text { Diabetes due to underlying } \\
\text { condition w diabetic polyneurop }\end{array}$ & 20437 & 0.1237 & 0.0488 & 2.5346 & 2.3283 & 2.7591 \\
\hline J93 & J93.83 & Other pneumothorax & 1123 & 0.1345 & 0.0531 & 2.5341 & 1.8406 & 3.4889 \\
\hline A40 & A40.9 & Streptococcal sepsis, unspecified & 789 & 0.1343 & 0.0531 & 2.5281 & 1.7269 & 3.7010 \\
\hline C67 & C67.9 & $\begin{array}{l}\text { Malignant neoplasm of bladder, } \\
\text { unspecified }\end{array}$ & 4135 & 0.1323 & 0.0524 & 2.5266 & 2.1310 & 2.9956 \\
\hline C18 & C18.9 & $\begin{array}{l}\text { Malignant neoplasm of colon, } \\
\text { unspecified }\end{array}$ & 4296 & 0.1318 & 0.0523 & 2.5179 & 2.1295 & 2.9771 \\
\hline T44 & $\begin{array}{l}\text { T44.7X5 } \\
\text { A }\end{array}$ & $\begin{array}{l}\text { Adverse effect of beta- } \\
\text { adrenoreceptor antagonists, init }\end{array}$ & 831 & 0.1336 & 0.0531 & 2.5139 & 1.7318 & 3.6493 \\
\hline R27 & R27.0 & Ataxia, unspecified & 5819 & 0.1306 & 0.0520 & 2.5125 & 2.1722 & 2.9061 \\
\hline Z93 & Z93.3 & Colostomy status & 4186 & 0.1312 & 0.0524 & 2.5048 & 2.1131 & 2.9692 \\
\hline Z89 & Z89.519 & $\begin{array}{l}\text { Acquired absence of unspecified } \\
\text { leg below knee }\end{array}$ & 5730 & 0.1302 & 0.0520 & 2.5031 & 2.1612 & 2.8992 \\
\hline Z43 & Z43.3 & $\begin{array}{l}\text { Encounter for attention to } \\
\text { colostomy }\end{array}$ & 2992 & 0.1317 & 0.0526 & 2.5019 & 2.0489 & 3.0550 \\
\hline E10 & E10.9 & $\begin{array}{l}\text { Type } 1 \text { diabetes mellitus without } \\
\text { complications }\end{array}$ & 20517 & 0.1220 & 0.0489 & 2.4951 & 2.2911 & 2.7172 \\
\hline H35 & H35.31 & $\begin{array}{l}\text { Nonexudative age-related } \\
\text { macular degeneration }\end{array}$ & 60393 & 0.1051 & 0.0421 & 2.4933 & 2.3418 & 2.6547 \\
\hline 138 & 138. & Endocarditis, valve unspecified & 2607 & 0.1312 & 0.0527 & 2.4880 & 2.0084 & 3.0820 \\
\hline 124 & 124.8 & $\begin{array}{l}\text { Other forms of acute ischemic } \\
\text { heart disease }\end{array}$ & 3221 & 0.1307 & 0.0526 & 2.4854 & 2.0481 & 3.0160 \\
\hline $\mathrm{C} 25$ & C25.9 & $\begin{array}{l}\text { Malignant neoplasm of pancreas, } \\
\text { unspecified }\end{array}$ & 636 & 0.1321 & 0.0532 & 2.4835 & 1.6181 & 3.8117 \\
\hline 177 & 177.1 & Stricture of artery & 7515 & 0.1280 & 0.0516 & 2.4789 & 2.1759 & 2.8241 \\
\hline R32 & R32. & Unspecified urinary incontinence & 18819 & 0.1220 & 0.0493 & 2.4746 & 2.2651 & 2.7034 \\
\hline 166 & 166.9 & $\begin{array}{l}\text { Occlusion and stenosis of } \\
\text { unspecified cerebral artery }\end{array}$ & 883 & 0.1314 & 0.0531 & 2.4728 & 1.7165 & 3.5623 \\
\hline G12 & G12.21 & Amyotrophic lateral sclerosis & 685 & 0.1314 & 0.0532 & 2.4710 & 1.6331 & 3.7387 \\
\hline 142 & 142.9 & Cardiomyopathy, unspecified & 15337 & 0.1234 & 0.0500 & 2.4677 & 2.2418 & 2.7164 \\
\hline 172 & 172.3 & Aneurysm of iliac artery & 3524 & 0.1294 & 0.0525 & 2.4632 & 2.0446 & 2.9675 \\
\hline 105 & 105.8 & $\begin{array}{l}\text { Other rheumatic mitral valve } \\
\text { diseases }\end{array}$ & 1041 & 0.1306 & 0.0531 & 2.4607 & 1.7558 & 3.4485 \\
\hline F09 & F09. & $\begin{array}{l}\text { Unsp mental disorder due to } \\
\text { known physiological condition }\end{array}$ & 2150 & 0.1298 & 0.0528 & 2.4557 & 1.9380 & 3.1118 \\
\hline R77 & $\mathrm{R} 77.0$ & Abnormality of albumin & 1284 & 0.1301 & 0.0530 & 2.4522 & 1.8076 & 3.3266 \\
\hline D62 & D62. & Acute posthemorrhagic anemia & 6406 & 0.1266 & 0.0519 & 2.4383 & 2.1167 & 2.8087 \\
\hline M86 & M86.9 & Osteomyelitis, unspecified & 7523 & 0.1252 & 0.0517 & 2.4219 & 2.1227 & 2.7632 \\
\hline $\mathrm{J43}$ & J43.9 & Emphysema, unspecified & 9950 & 0.1238 & 0.0512 & 2.4182 & 2.1523 & 2.7169 \\
\hline 187 & 187.2 & $\begin{array}{l}\text { Venous insufficiency (chronic) } \\
\text { (peripheral) }\end{array}$ & 23285 & 0.1175 & 0.0486 & 2.4162 & 2.2250 & 2.6239 \\
\hline M81 & M81.0 & $\begin{array}{l}\text { Age-related osteoporosis w/o } \\
\text { current pathological fracture }\end{array}$ & 10016 & 0.1236 & 0.0512 & 2.4143 & 2.1494 & 2.7118 \\
\hline K72 & K72.90 & $\begin{array}{l}\text { Hepatic failure, unspecified } \\
\text { without coma }\end{array}$ & 1867 & 0.1275 & 0.0529 & 2.4089 & 1.8641 & 3.1128 \\
\hline W19 & $\begin{array}{c}\text { W19.XX } \\
\text { XA }\end{array}$ & Unspecified fall, initial encounter & 21689 & 0.1178 & 0.0489 & 2.4085 & 2.2128 & 2.6214 \\
\hline D38 & D38.1 & $\begin{array}{l}\text { Neoplasm of uncertain behavior } \\
\text { of trachea, bronchus and lung }\end{array}$ & 1841 & 0.1271 & 0.0529 & 2.4015 & 1.8543 & 3.1101 \\
\hline
\end{tabular}


medRxiv preprint doi: https://doi.org/10.1101/2022.01.22.22269694; this version posted February 21, 2022. The copyright holder for this preprint (which was not certified by peer review) is the author/funder, who has granted medRxiv a license to display the preprint in perpetuity. This article is a US Government work. It is not subject to copyright under 17 USC 105 and is also made available for use under a CCO license.

\begin{tabular}{|c|c|c|c|c|c|c|c|c|}
\hline T83 & $\begin{array}{c}\text { T83.098 } \\
\text { A }\end{array}$ & $\begin{array}{l}\text { Mech compl of other urinary } \\
\text { catheter, initial encounter }\end{array}$ & 3957 & 0.1259 & 0.0525 & 2.3983 & 2.0056 & 2.8679 \\
\hline 107 & 107.1 & Rheumatic tricuspid insufficiency & 3276 & 0.1258 & 0.0526 & 2.3898 & 1.9643 & 2.9075 \\
\hline M31 & M31.6 & Other giant cell arteritis & 1113 & 0.1267 & 0.0531 & 2.3863 & 1.7117 & 3.3268 \\
\hline T38 & $\begin{array}{c}\text { T38.0X5 } \\
A\end{array}$ & $\begin{array}{l}\text { Adverse effect of glucocort/synth } \\
\text { analog, init }\end{array}$ & 2095 & 0.1260 & 0.0529 & 2.3831 & 1.8674 & 3.0411 \\
\hline E09 & E09.65 & $\begin{array}{l}\text { Drug or chemical induced } \\
\text { diabetes mellitus w } \\
\text { hyperglycemia }\end{array}$ & 893 & 0.1265 & 0.0531 & 2.3814 & 1.6435 & 3.4506 \\
\hline E63 & E63.9 & $\begin{array}{l}\text { Nutritional deficiency, } \\
\text { unspecified }\end{array}$ & 1355 & 0.1255 & 0.0530 & 2.3654 & 1.7469 & 3.2027 \\
\hline $\mathrm{H} 90$ & $\mathrm{H} 90.3$ & $\begin{array}{l}\text { Sensorineural hearing loss, } \\
\text { bilateral }\end{array}$ & 118138 & 0.0855 & 0.0362 & 2.3616 & 2.2218 & 2.5102 \\
\hline 161 & 161.9 & $\begin{array}{l}\text { Nontraumatic intracerebral } \\
\text { hemorrhage, unspecified }\end{array}$ & 1197 & 0.1253 & 0.0531 & 2.3610 & 1.7102 & 3.2595 \\
\hline G46 & G46.4 & Cerebellar stroke syndrome & 4838 & 0.1234 & 0.0523 & 2.3586 & 2.0018 & 2.7791 \\
\hline R13 & R13.10 & Dysphagia, unspecified & 40814 & 0.1080 & 0.0459 & 2.3554 & 2.1977 & 2.5245 \\
\hline $\mathrm{C} 20$ & C20. & Malignant neoplasm of rectum & 935 & 0.1251 & 0.0531 & 2.3552 & 1.6352 & 3.3920 \\
\hline E21 & E21.3 & $\begin{array}{l}\text { Hyperparathyroidism, } \\
\text { unspecified }\end{array}$ & 4412 & 0.1233 & 0.0524 & 2.3526 & 1.9817 & 2.7930 \\
\hline K94 & K94.23 & Gastrostomy malfunction & 1165 & 0.1245 & 0.0531 & 2.3446 & 1.6888 & 3.2551 \\
\hline S37 & $\begin{array}{c}\text { S37.009 } \\
\text { A }\end{array}$ & $\begin{array}{l}\text { Unspecified injury of unspecified } \\
\text { kidney, initial encounter }\end{array}$ & 989 & 0.1244 & 0.0531 & 2.3412 & 1.6399 & 3.3423 \\
\hline S81 & $\begin{array}{l}\text { S81.009 } \\
\text { A }\end{array}$ & $\begin{array}{l}\text { Unspecified open wound, } \\
\text { unspecified knee, initial } \\
\text { encounter }\end{array}$ & 8362 & 0.1208 & 0.0516 & 2.3395 & 2.0586 & 2.6588 \\
\hline D89 & D89.9 & $\begin{array}{l}\text { Disorder involving the immune } \\
\text { mechanism, unspecified }\end{array}$ & 1590 & 0.1239 & 0.0530 & 2.3378 & 1.7633 & 3.0995 \\
\hline G45 & G45.9 & $\begin{array}{l}\text { Transient cerebral ischemic } \\
\text { attack, unspecified }\end{array}$ & 14740 & 0.1178 & 0.0504 & 2.3365 & 2.1140 & 2.5823 \\
\hline Y84 & Y84.8 & $\begin{array}{l}\text { Oth medical procedures cause } \\
\text { abn react/compl, w/o misadvnt }\end{array}$ & 3576 & 0.1228 & 0.0526 & 2.3343 & 1.9295 & 2.8240 \\
\hline R47 & R47.1 & Dysarthria and anarthria & 8462 & 0.1204 & 0.0516 & 2.3330 & 2.0539 & 2.6500 \\
\hline 145 & 145.10 & $\begin{array}{l}\text { Unspecified right bundle-branch } \\
\text { block }\end{array}$ & 15283 & 0.1173 & 0.0503 & 2.3302 & 2.1113 & 2.5718 \\
\hline G81 & G81.90 & $\begin{array}{l}\text { Hemiplegia, unspecified affecting } \\
\text { unspecified side }\end{array}$ & 3445 & 0.1225 & 0.0526 & 2.3279 & 1.9171 & 2.8267 \\
\hline R41 & $\mathrm{R} 41.2$ & Retrograde amnesia & 42168 & 0.1065 & 0.0458 & 2.3270 & 2.1720 & 2.4930 \\
\hline 111 & I11.9 & $\begin{array}{l}\text { Hypertensive heart disease } \\
\text { without heart failure }\end{array}$ & 28332 & 0.1117 & 0.0480 & 2.3255 & 2.1510 & 2.5142 \\
\hline Q84 & Q84.5 & Enlarged and hypertrophic nails & 1698 & 0.1231 & 0.0530 & 2.3233 & 1.7664 & 3.0557 \\
\hline Q27 & Q27.9 & $\begin{array}{l}\text { Congenital malformation of } \\
\text { peripheral vascular system, unsp }\end{array}$ & 1138 & 0.1230 & 0.0531 & 2.3170 & 1.6589 & 3.2362 \\
\hline $\mathrm{J} 12$ & $\mathrm{~J} 12.9$ & Viral pneumonia, unspecified & 1835 & 0.1226 & 0.0530 & 2.3155 & 1.7777 & 3.0160 \\
\hline T36 & $\begin{array}{c}\text { T36.8X5 } \\
\mathrm{A} \\
\end{array}$ & $\begin{array}{l}\text { Adverse effect of other systemic } \\
\text { antibiotics, init encntr }\end{array}$ & 1254 & 0.1228 & 0.0531 & 2.3139 & 1.6824 & 3.1826 \\
\hline 199 & 199.8 & $\begin{array}{l}\text { Other disorder of circulatory } \\
\text { system }\end{array}$ & 4579 & 0.1212 & 0.0524 & 2.3130 & 1.9511 & 2.7419 \\
\hline S32 & $\begin{array}{c}\text { S32.009 } \\
\text { A }\end{array}$ & $\begin{array}{l}\text { Unsp fracture of unsp lumbar } \\
\text { vertebra, init for clos fx }\end{array}$ & 4732 & 0.1211 & 0.0524 & 2.3121 & 1.9555 & 2.7338 \\
\hline J95 & J95.811 & Postprocedural pneumothorax & 2669 & 0.1218 & 0.0528 & 2.3067 & 1.8499 & 2.8763 \\
\hline D69 & D69.6 & Thrombocytopenia, unspecified & 15284 & 0.1161 & 0.0504 & 2.3056 & 2.0880 & 2.5458 \\
\hline Z51 & Z51.89 & $\begin{array}{l}\text { Encounter for other specified } \\
\text { aftercare }\end{array}$ & 178296 & 0.0729 & 0.0317 & 2.3027 & 2.1516 & 2.4644 \\
\hline Z99 & Z99.89 & $\begin{array}{l}\text { Dependence on other enabling } \\
\text { machines and devices }\end{array}$ & 21756 & 0.1133 & 0.0492 & 2.3027 & 2.1125 & 2.5100 \\
\hline N05 & N05.9 & $\begin{array}{l}\text { Unsp nephritic syndrome with } \\
\text { unspecified morphologic } \\
\text { changes }\end{array}$ & 1450 & 0.1221 & 0.0530 & 2.3017 & 1.7091 & 3.0996 \\
\hline
\end{tabular}


medRxiv preprint doi: https://doi.org/10.1101/2022.01.22.22269694; this version posted February 21, 2022. The copyright holder for this preprint (which was not certified by peer review) is the author/funder, who has granted medRxiv a license to display the preprint in perpetuity. This article is a US Government work. It is not subject to copyright under 17 USC 105 and is also made available for use under a CCO license.

\begin{tabular}{|c|c|c|c|c|c|c|c|c|}
\hline N03 & N03.9 & $\begin{array}{l}\text { Chronic nephritic syndrome with } \\
\text { unsp morphologic changes }\end{array}$ & 736 & 0.1223 & 0.0532 & 2.2994 & 1.5164 & 3.4866 \\
\hline R34 & R34. & Anuria and oliguria & 720 & 0.1222 & 0.0532 & 2.2981 & 1.5085 & 3.5011 \\
\hline D09 & D09.0 & Carcinoma in situ of bladder & 3148 & 0.1210 & 0.0527 & 2.2967 & 1.8724 & 2.8172 \\
\hline C76 & C76.0 & $\begin{array}{l}\text { Malignant neoplasm of head, } \\
\text { face and neck }\end{array}$ & 3221 & 0.1208 & 0.0527 & 2.2923 & 1.8726 & 2.8061 \\
\hline 137 & 137.0 & $\begin{array}{l}\text { Nonrheumatic pulmonary valve } \\
\text { stenosis }\end{array}$ & 1552 & 0.1211 & 0.0530 & 2.2847 & 1.7112 & 3.0505 \\
\hline Z94 & Z94.0 & Kidney transplant status & 3033 & 0.1203 & 0.0527 & 2.2824 & 1.8526 & 2.8120 \\
\hline J16 & J16.8 & $\begin{array}{l}\text { Pneumonia due to other } \\
\text { specified infectious organisms }\end{array}$ & 1240 & 0.1210 & 0.0531 & 2.2789 & 1.6494 & 3.1486 \\
\hline A04 & A04.7 & $\begin{array}{l}\text { Enterocolitis due to Clostridium } \\
\text { difficile }\end{array}$ & 5175 & 0.1190 & 0.0523 & 2.2754 & 1.9351 & 2.6755 \\
\hline$\overline{Z 85}$ & Z85.828 & $\begin{array}{l}\text { Personal history of other } \\
\text { malignant neoplasm of skin }\end{array}$ & 43622 & 0.1042 & 0.0458 & 2.2743 & 2.1233 & 2.4362 \\
\hline L60 & L60.0 & Ingrowing nail & 53817 & 0.1007 & 0.0444 & 2.2688 & 2.1255 & 2.4219 \\
\hline H59 & H59.032 & $\begin{array}{l}\text { Cystoid macular edema following } \\
\text { cataract surgery, left eye }\end{array}$ & 2323 & 0.1197 & 0.0529 & 2.2634 & 1.7830 & 2.8732 \\
\hline C77 & $\mathrm{C} 77.0$ & $\begin{array}{l}\text { Sec and unsp malig neoplasm of } \\
\text { nodes of head, face and neck }\end{array}$ & 2569 & 0.1195 & 0.0528 & 2.2622 & 1.8024 & 2.8393 \\
\hline R53 & R53.1 & Weakness & 65148 & 0.0971 & 0.0430 & 2.2599 & 2.1221 & 2.4067 \\
\hline E86 & E86.0 & Dehydration & 28085 & 0.1091 & 0.0483 & 2.2594 & 2.0876 & 2.4453 \\
\hline T17 & $\begin{array}{c}\mathrm{T} 17.300 \\
\mathrm{~A}\end{array}$ & $\begin{array}{l}\text { Unsp foreign body in larynx } \\
\text { causing asphyxiation, init }\end{array}$ & 842 & 0.1200 & 0.0532 & 2.2563 & 1.5221 & 3.3445 \\
\hline $\mathrm{R} 80$ & $\mathrm{R} 80.3$ & Bence Jones proteinuria & 13651 & 0.1142 & 0.0508 & 2.2489 & 2.0245 & 2.4983 \\
\hline Z79 & Z79.899 & $\begin{array}{l}\text { Other long term (current) drug } \\
\text { therapy }\end{array}$ & 147374 & 0.0777 & 0.0346 & 2.2438 & 2.1065 & 2.3899 \\
\hline D47 & D47.2 & Monoclonal gammopathy & 5592 & 0.1166 & 0.0523 & 2.2306 & 1.9049 & 2.6119 \\
\hline N31 & N31.9 & $\begin{array}{l}\text { Neuromuscular dysfunction of } \\
\text { bladder, unspecified }\end{array}$ & 7306 & 0.1158 & 0.0520 & 2.2287 & 1.9386 & 2.5621 \\
\hline D50 & D50.9 & $\begin{array}{l}\text { Iron deficiency anemia, } \\
\text { unspecified }\end{array}$ & 31287 & 0.1065 & 0.0479 & 2.2208 & 2.0569 & 2.3978 \\
\hline S51 & $\begin{array}{c}\text { S51.809 } \\
\text { A }\end{array}$ & $\begin{array}{l}\text { Unspecified open wound of } \\
\text { unspecified forearm, init encntr }\end{array}$ & 3177 & 0.1171 & 0.0527 & 2.2207 & 1.8053 & 2.7317 \\
\hline R15 & R15.9 & Full incontinence of feces & 3962 & 0.1166 & 0.0526 & 2.2176 & 1.8405 & 2.6720 \\
\hline 134 & 134.0 & $\begin{array}{l}\text { Nonrheumatic mitral (valve) } \\
\text { insufficiency }\end{array}$ & 11109 & 0.1137 & 0.0513 & 2.2167 & 1.9744 & 2.4887 \\
\hline 189 & 189.0 & $\begin{array}{l}\text { Lymphedema, not elsewhere } \\
\text { classified }\end{array}$ & 5047 & 0.1161 & 0.0524 & 2.2165 & 1.8772 & 2.6172 \\
\hline $\mathrm{H} 54$ & $\mathrm{H} 54.7$ & Unspecified visual loss & 25803 & 0.1080 & 0.0488 & 2.2108 & 2.0363 & 2.4001 \\
\hline 115 & 115.8 & Other secondary hypertension & 4169 & 0.1161 & 0.0526 & 2.2092 & 1.8411 & 2.6510 \\
\hline R58 & R58. & $\begin{array}{l}\text { Hemorrhage, not elsewhere } \\
\text { classified }\end{array}$ & 1357 & 0.1172 & 0.0531 & 2.2077 & 1.6115 & 3.0243 \\
\hline D01 & D01.0 & Carcinoma in situ of colon & 2376 & 0.1166 & 0.0529 & 2.2045 & 1.7352 & 2.8007 \\
\hline R63 & R63.4 & Abnormal weight loss & 34666 & 0.1046 & 0.0475 & 2.2026 & 2.0448 & 2.3726 \\
\hline 136 & 136.1 & $\begin{array}{l}\text { Nonrheumatic tricuspid (valve) } \\
\text { insufficiency }\end{array}$ & 3914 & 0.1157 & 0.0526 & 2.2002 & 1.8226 & 2.6561 \\
\hline J13 & J13. & $\begin{array}{l}\text { Pneumonia due to Streptococcus } \\
\text { pneumoniae }\end{array}$ & 1115 & 0.1166 & 0.0531 & 2.1948 & 1.5499 & 3.1081 \\
\hline N28 & N28.9 & $\begin{array}{l}\text { Disorder of kidney and ureter, } \\
\text { unspecified }\end{array}$ & 27416 & 0.1067 & 0.0486 & 2.1941 & 2.0241 & 2.3783 \\
\hline 143 & 143. & $\begin{array}{l}\text { Cardiomyopathy in diseases } \\
\text { classified elsewhere }\end{array}$ & 1050 & 0.1162 & 0.0531 & 2.1867 & 1.5269 & 3.1315 \\
\hline D68 & D68.8 & $\begin{array}{l}\text { Other specified coagulation } \\
\text { defects }\end{array}$ & 5690 & 0.1142 & 0.0523 & 2.1845 & 1.8648 & 2.5591 \\
\hline E83 & E83.42 & Hypomagnesemia & 22641 & 0.1079 & 0.0494 & 2.1827 & 2.0014 & 2.3806 \\
\hline E16 & E16.2 & Hypoglycemia, unspecified & 9251 & 0.1126 & 0.0517 & 2.1799 & 1.9207 & 2.4742 \\
\hline C44 & C44.91 & $\begin{array}{l}\text { Basal cell carcinoma of skin, } \\
\text { unspecified }\end{array}$ & 21770 & 0.1080 & 0.0496 & 2.1778 & 1.9940 & 2.3785 \\
\hline $\mathrm{H} 61$ & H61.23 & Impacted cerumen, bilateral & 58221 & 0.0966 & 0.0444 & 2.1776 & 2.0411 & 2.3232 \\
\hline
\end{tabular}


medRxiv preprint doi: https://doi.org/10.1101/2022.01.22.22269694; this version posted February 21, 2022. The copyright holder for this preprint (which was not certified by peer review) is the author/funder, who has granted medRxiv a license to display the preprint in perpetuity. This article is a US Government work. It is not subject to copyright under 17 USC 105 and is also made available for use under a CCO license.

\begin{tabular}{|c|c|c|c|c|c|c|c|c|}
\hline 151 & 151.7 & Cardiomegaly & 24480 & 0.1066 & 0.0492 & 2.1674 & 1.9918 & 2.3584 \\
\hline Z46 & Z46.1 & $\begin{array}{l}\text { Encounter for fitting and } \\
\text { adjustment of hearing aid }\end{array}$ & 98123 & 0.0863 & 0.0399 & 2.1625 & 2.0355 & 2.2975 \\
\hline H52 & H52.4 & Presbyopia & 192666 & 0.0694 & 0.0323 & 2.1442 & 2.0003 & 2.2985 \\
\hline D41 & D41.4 & $\begin{array}{l}\text { Neoplasm of uncertain behavior } \\
\text { of bladder }\end{array}$ & 3097 & 0.1130 & 0.0528 & 2.1412 & 1.7289 & 2.6517 \\
\hline E20 & E20.1 & Pseudohypoparathyroidism & 1647 & 0.1135 & 0.0530 & 2.1408 & 1.6002 & 2.8640 \\
\hline C61 & C61. & Malignant neoplasm of prostate & 19707 & 0.1069 & 0.0500 & 2.1359 & 1.9473 & 2.3427 \\
\hline K55 & K55.20 & $\begin{array}{l}\text { Angiodysplasia of colon without } \\
\text { hemorrhage }\end{array}$ & 2499 & 0.1128 & 0.0529 & 2.1336 & 1.6821 & 2.7064 \\
\hline B96 & B96.81 & $\begin{array}{l}\text { Helicobacter pylori as the cause } \\
\text { of diseases classd elswhr }\end{array}$ & 20262 & 0.1066 & 0.0500 & 2.1330 & 1.9466 & 2.3373 \\
\hline B95 & B95.61 & $\begin{array}{l}\text { Methicillin suscep staph infct } \\
\text { causing dis classd elswhr }\end{array}$ & 13437 & 0.1086 & 0.0511 & 2.1267 & 1.9078 & 2.3708 \\
\hline N32 & N32.81 & Overactive bladder & 19165 & 0.1063 & 0.0502 & 2.1188 & 1.9291 & 2.3272 \\
\hline E13 & E13.9 & $\begin{array}{l}\text { Other specified diabetes mellitus } \\
\text { without complications }\end{array}$ & 11229 & 0.1089 & 0.0514 & 2.1177 & 1.8824 & 2.3824 \\
\hline T85 & $\begin{array}{c}\text { T85.398 } \\
\text { A }\end{array}$ & $\begin{array}{l}\text { Mech compl of ocular prosth } \\
\text { dev/grft, init }\end{array}$ & 3710 & 0.1113 & 0.0527 & 2.1128 & 1.7342 & 2.5739 \\
\hline 185 & 185.00 & $\begin{array}{l}\text { Esophageal varices without } \\
\text { bleeding }\end{array}$ & 2480 & 0.1117 & 0.0529 & 2.1114 & 1.6608 & 2.6842 \\
\hline W18 & $\begin{array}{l}\text { W18.49X } \\
A\end{array}$ & $\begin{array}{l}\text { Oth slipping, tripping and } \\
\text { stumbling w/o falling, init }\end{array}$ & 13407 & 0.1077 & 0.0511 & 2.1079 & 1.8898 & 2.3511 \\
\hline R29 & R29.6 & Repeated falls & 19639 & 0.1055 & 0.0501 & 2.1035 & 1.9164 & 2.3089 \\
\hline $\mathrm{H} 02$ & H02.839 & $\begin{array}{l}\text { Dermatochalasis of unspecified } \\
\text { eye, unspecified eyelid }\end{array}$ & 29211 & 0.1024 & 0.0487 & 2.1019 & 1.9401 & 2.2772 \\
\hline B35 & B35.1 & Tinea unguium & 95767 & 0.0854 & 0.0407 & 2.0963 & 1.9729 & 2.2274 \\
\hline G99 & G99.0 & $\begin{array}{l}\text { Autonomic neuropathy in } \\
\text { diseases classified elsewhere }\end{array}$ & 4227 & 0.1100 & 0.0526 & 2.0908 & 1.7351 & 2.5194 \\
\hline K26 & K26.9 & $\begin{array}{l}\text { Duodenal ulcer, unsp as acute or } \\
\text { chronic, w/o hemor or perf }\end{array}$ & 3428 & 0.1103 & 0.0527 & 2.0904 & 1.7008 & 2.5693 \\
\hline 131 & I31.9 & $\begin{array}{l}\text { Disease of pericardium, } \\
\text { unspecified }\end{array}$ & 3328 & 0.1103 & 0.0528 & 2.0899 & 1.6953 & 2.5764 \\
\hline $\mathrm{H} 49$ & $\mathrm{H} 49.20$ & $\begin{array}{l}\text { Sixth [abducent] nerve palsy, } \\
\text { unspecified eye }\end{array}$ & 1988 & 0.1107 & 0.0530 & 2.0883 & 1.5957 & 2.7330 \\
\hline T87 & T87.89 & $\begin{array}{l}\text { Other complications of } \\
\text { amputation stump }\end{array}$ & 1707 & 0.1107 & 0.0530 & 2.0875 & 1.5620 & 2.7898 \\
\hline L62 & L62. & $\begin{array}{l}\text { Nail disorders in diseases } \\
\text { classified elsewhere }\end{array}$ & 1530 & 0.1105 & 0.0531 & 2.0813 & 1.5318 & 2.8279 \\
\hline N47 & N47.6 & Balanoposthitis & 6273 & 0.1086 & 0.0523 & 2.0761 & 1.7776 & 2.4247 \\
\hline N39 & N39.0 & $\begin{array}{l}\text { Urinary tract infection, site not } \\
\text { specified }\end{array}$ & 64087 & 0.0920 & 0.0444 & 2.0733 & 1.9446 & 2.2105 \\
\hline T46 & $\begin{array}{c}\text { T46.4X5 } \\
\text { A }\end{array}$ & $\begin{array}{l}\text { Adverse effect of angiotens- } \\
\text { convert-enzyme inhibitors, init }\end{array}$ & 1931 & 0.1098 & 0.0530 & 2.0712 & 1.5746 & 2.7244 \\
\hline Z16 & Z16.11 & Resistance to penicillins & 2546 & 0.1092 & 0.0529 & 2.0638 & 1.6235 & 2.6234 \\
\hline 182 & 182.409 & $\begin{array}{l}\text { Acute embolism and thombos } \\
\text { unsp deep vn unsp lower } \\
\text { extremity }\end{array}$ & 14028 & 0.1046 & 0.0511 & 2.0456 & 1.8351 & 2.2803 \\
\hline N12 & N12. & $\begin{array}{l}\text { Tubulo-interstitial nephritis, not } \\
\text { spcf as acute or chronic }\end{array}$ & 1965 & 0.1084 & 0.0530 & 2.0449 & 1.5552 & 2.6887 \\
\hline S91 & $\begin{array}{c}\text { S91.309 } \\
\text { A }\end{array}$ & $\begin{array}{l}\text { Unspecified open wound, } \\
\text { unspecified foot, initial encounter }\end{array}$ & 11184 & 0.1054 & 0.0516 & 2.0447 & 1.8135 & 2.3054 \\
\hline $\mathrm{H} 21$ & $\mathrm{H} 21.81$ & Floppy iris syndrome & 5158 & 0.1072 & 0.0525 & 2.0422 & 1.7199 & 2.4248 \\
\hline $\mathrm{H} 01$ & H01.009 & $\begin{array}{l}\text { Unspecified blepharitis } \\
\text { unspecified eye, unspecified } \\
\text { eyelid }\end{array}$ & 31384 & 0.0992 & 0.0487 & 2.0391 & 1.8843 & 2.2066 \\
\hline 197 & 197.710 & $\begin{array}{l}\text { Intraoperative cardiac arrest } \\
\text { during cardiac surgery }\end{array}$ & 2682 & 0.1078 & 0.0529 & 2.0371 & 1.6095 & 2.5783 \\
\hline L85 & L85.3 & Xerosis cutis & 31066 & 0.0991 & 0.0487 & 2.0350 & 1.8799 & 2.2029 \\
\hline M15 & M15.9 & Polyosteoarthritis, unspecified & 70950 & 0.0892 & 0.0439 & 2.0333 & 1.9090 & 2.1656 \\
\hline
\end{tabular}


medRxiv preprint doi: https://doi.org/10.1101/2022.01.22.22269694; this version posted February 21, 2022. The copyright holder for this preprint (which was not certified by peer review) is the author/funder, who has granted medRxiv a license to display the preprint in perpetuity. This article is a US Government work. It is not subject to copyright under 17 USC 105 and is also made available for use under a CCO license.

\begin{tabular}{|c|c|c|c|c|c|c|c|c|}
\hline 120 & 120.8 & Other forms of angina pectoris & 31188 & 0.0990 & 0.0487 & 2.0318 & 1.8770 & 2.1993 \\
\hline N13 & $\mathrm{N} 13.30$ & Unspecified hydronephrosis & 13082 & 0.1040 & 0.0513 & 2.0264 & 1.8109 & 2.2676 \\
\hline L57 & L57.0 & Actinic keratosis & 46813 & 0.0945 & 0.0467 & 2.0227 & 1.8866 & 2.1685 \\
\hline G60 & G60.9 & $\begin{array}{l}\text { Hereditary and idiopathic } \\
\text { neuropathy, unspecified }\end{array}$ & 30297 & 0.0988 & 0.0489 & 2.0219 & 1.8661 & 2.1907 \\
\hline M10 & M10.9 & Gout, unspecified & 32778 & 0.0981 & 0.0485 & 2.0210 & 1.8693 & 2.1849 \\
\hline M1A & $\begin{array}{c}\text { M1A.9XX } \\
0\end{array}$ & $\begin{array}{l}\text { Chronic gout, unspecified, } \\
\text { without tophus (tophi) }\end{array}$ & 5350 & 0.1060 & 0.0525 & 2.0192 & 1.7039 & 2.3928 \\
\hline 126 & 126.99 & $\begin{array}{l}\text { Other pulmonary embolism } \\
\text { without acute cor pulmonale }\end{array}$ & 7323 & 0.1051 & 0.0522 & 2.0148 & 1.7400 & 2.3330 \\
\hline K56 & K56.60 & Unspecified intestinal obstruction & 8535 & 0.1047 & 0.0520 & 2.0141 & 1.7569 & 2.3090 \\
\hline G58 & G58.9 & Mononeuropathy, unspecified & 20738 & 0.1011 & 0.0502 & 2.0134 & 1.8348 & 2.2093 \\
\hline D30 & D30.3 & Benign neoplasm of bladder & 1931 & 0.1067 & 0.0530 & 2.0119 & 1.5228 & 2.6581 \\
\hline K59 & K59.00 & Constipation, unspecified & 59540 & 0.0911 & 0.0453 & 2.0108 & 1.8833 & 2.1469 \\
\hline R01 & R01.1 & Cardiac murmur, unspecified & 14466 & 0.1028 & 0.0511 & 2.0104 & 1.8045 & 2.2398 \\
\hline 147 & 147.1 & Supraventricular tachycardia & 12549 & 0.1031 & 0.0514 & 2.0053 & 1.7873 & 2.2500 \\
\hline L11 & L11.0 & Acquired keratosis follicularis & 16439 & 0.1020 & 0.0509 & 2.0047 & 1.8095 & 2.2209 \\
\hline 149 & 149.9 & Cardiac arrhythmia, unspecified & 59805 & 0.0907 & 0.0453 & 2.0015 & 1.8746 & 2.1370 \\
\hline M62 & M62.81 & Muscle weakness (generalized) & 71400 & 0.0882 & 0.0441 & 2.0014 & 1.8789 & 2.1319 \\
\hline G82 & G82.20 & Paraplegia, unspecified & 2430 & 0.1058 & 0.0530 & 1.9973 & 1.5557 & 2.5643 \\
\hline J98 & $\mathrm{J} 98.4$ & Other disorders of lung & 35483 & 0.0964 & 0.0483 & 1.9959 & 1.8494 & 2.1540 \\
\hline C64 & C64.9 & $\begin{array}{l}\text { Malignant neoplasm of unsp } \\
\text { kidney, except renal pelvis }\end{array}$ & 3149 & 0.1048 & 0.0528 & 1.9829 & 1.5896 & 2.4736 \\
\hline H91 & H91.90 & $\begin{array}{l}\text { Unspecified hearing loss, } \\
\text { unspecified ear }\end{array}$ & 71156 & 0.0876 & 0.0443 & 1.9790 & 1.8576 & 2.1083 \\
\hline E53 & E53.8 & $\begin{array}{l}\text { Deficiency of other specified B } \\
\text { group vitamins }\end{array}$ & 19291 & 0.0996 & 0.0505 & 1.9712 & 1.7899 & 2.1710 \\
\hline R44 & R44.0 & Auditory hallucinations & 4298 & 0.1038 & 0.0527 & 1.9697 & 1.6273 & 2.3841 \\
\hline R97 & R97.2 & $\begin{array}{l}\text { Elevated prostate specific } \\
\text { antigen [PSA] }\end{array}$ & 36803 & 0.0950 & 0.0483 & 1.9694 & 1.8259 & 2.1241 \\
\hline R91 & R91.8 & $\begin{array}{l}\text { Other nonspecific abnormal } \\
\text { finding of lung field }\end{array}$ & 37585 & 0.0947 & 0.0482 & 1.9658 & 1.8235 & 2.1193 \\
\hline $\mathrm{H} 40$ & H40.009 & $\begin{array}{l}\text { Preglaucoma, unspecified, } \\
\text { unspecified eye }\end{array}$ & 61759 & 0.0889 & 0.0454 & 1.9583 & 1.8344 & 2.0905 \\
\hline 116 & 116.0 & Hypertensive urgency & 5096 & 0.1024 & 0.0526 & 1.9481 & 1.6320 & 2.3254 \\
\hline$\overline{Q 61}$ & Q61.00 & $\begin{array}{l}\text { Congenital renal cyst, } \\
\text { unspecified }\end{array}$ & 7172 & 0.1018 & 0.0523 & 1.9467 & 1.6744 & 2.2634 \\
\hline D49 & $\mathrm{D} 49.2$ & $\begin{array}{l}\text { Neoplasm of unsp behavior of } \\
\text { bone, soft tissue, and skin }\end{array}$ & 14001 & 0.0998 & 0.0513 & 1.9437 & 1.7390 & 2.1726 \\
\hline Z76 & $Z 76.0$ & $\begin{array}{l}\text { Encounter for issue of repeat } \\
\text { prescription }\end{array}$ & 161461 & 0.0715 & 0.0369 & 1.9405 & 1.8214 & 2.0674 \\
\hline D07 & D07.5 & Carcinoma in situ of prostate & 9818 & 0.1006 & 0.0519 & 1.9381 & 1.7008 & 2.2086 \\
\hline K74 & K74.60 & Unspecified cirrhosis of liver & 8439 & 0.1010 & 0.0521 & 1.9372 & 1.6840 & 2.2285 \\
\hline K83 & K83.1 & Obstruction of bile duct & 3048 & 0.1024 & 0.0529 & 1.9355 & 1.5416 & 2.4301 \\
\hline Z86 & Z86.010 & $\begin{array}{l}\text { Personal history of colonic } \\
\text { polyps }\end{array}$ & 103774 & 0.0803 & 0.0415 & 1.9350 & 1.8211 & 2.0561 \\
\hline N42 & N42.9 & Disorder of prostate, unspecified & 10264 & 0.1003 & 0.0519 & 1.9328 & 1.7003 & 2.1972 \\
\hline Y83 & Y83.1 & $\begin{array}{l}\text { Implnt of artif int dev cause abn } \\
\text { react/compl, w/o misadvnt }\end{array}$ & 11053 & 0.0999 & 0.0518 & 1.9296 & 1.7044 & 2.1845 \\
\hline L84 & L84. & Corns and callosities & 31962 & 0.0946 & 0.0490 & 1.9284 & 1.7801 & 2.0891 \\
\hline R04 & R04.0 & Epistaxis & 14831 & 0.0988 & 0.0513 & 1.9272 & 1.7282 & 2.1491 \\
\hline D72 & D72.829 & $\begin{array}{l}\text { Elevated white blood cell count, } \\
\text { unspecified }\end{array}$ & 25660 & 0.0958 & 0.0499 & 1.9221 & 1.7619 & 2.0968 \\
\hline G93 & G93.3 & Postviral fatigue syndrome & 39656 & 0.0925 & 0.0482 & 1.9201 & 1.7824 & 2.0685 \\
\hline B02 & B02.9 & Zoster without complications & 16479 & 0.0976 & 0.0511 & 1.9118 & 1.7221 & 2.1225 \\
\hline Z92 & Z92.3 & Personal history of irradiation & 8961 & 0.0994 & 0.0521 & 1.9092 & 1.6643 & 2.1900 \\
\hline N30 & N30.00 & Acute cystitis without hematuria & 10151 & 0.0988 & 0.0519 & 1.9027 & 1.6710 & 2.1666 \\
\hline R78 & R78.89 & $\begin{array}{l}\text { Finding of oth substances, not } \\
\text { normally found in blood }\end{array}$ & 32503 & 0.0933 & 0.0491 & 1.9012 & 1.7551 & 2.0595 \\
\hline R55 & R55. & Syncope and collapse & 30812 & 0.0937 & 0.0493 & 1.9004 & 1.7516 & 2.0619 \\
\hline
\end{tabular}


medRxiv preprint doi: https://doi.org/10.1101/2022.01.22.22269694; this version posted February 21, 2022. The copyright holder for this preprint (which was not certified by peer review) is the author/funder, who has granted medRxiv a license to display the preprint in perpetuity. This article is a US Government work. It is not subject to copyright under 17 USC 105 and is also made available for use under a CCO license.

\begin{tabular}{|c|c|c|c|c|c|c|c|c|}
\hline E03 & E03.9 & Hypothyroidism, unspecified & 35891 & 0.0925 & 0.0487 & 1.9000 & 1.7588 & 2.0526 \\
\hline $\mathrm{HO}$ & $\mathrm{H} 04.123$ & $\begin{array}{l}\text { Dry eye syndrome of bilateral } \\
\text { lacrimal glands }\end{array}$ & 82128 & 0.0830 & 0.0439 & 1.8911 & 1.7770 & 2.0126 \\
\hline K27 & K27.9 & $\begin{array}{l}\text { Peptic ulc, site unsp, unsp as ac } \\
\text { or chr, w/o hemor or perf }\end{array}$ & 7919 & 0.0987 & 0.0522 & 1.8901 & 1.6332 & 2.1874 \\
\hline $\mathrm{Z60}$ & $\mathrm{Z60.2}$ & Problems related to living alone & 11811 & 0.0974 & 0.0517 & 1.8817 & 1.6657 & 2.1257 \\
\hline E27 & E27.9 & $\begin{array}{l}\text { Disorder of adrenal gland, } \\
\text { unspecified }\end{array}$ & 3916 & 0.0991 & 0.0528 & 1.8767 & 1.5290 & 2.3034 \\
\hline F06 & F06.30 & $\begin{array}{l}\text { Mood disorder due to known } \\
\text { physiological condition, unsp }\end{array}$ & 31408 & 0.0926 & 0.0493 & 1.8761 & 1.7295 & 2.0351 \\
\hline Z65 & Z65.9 & $\begin{array}{l}\text { Problem related to unspecified } \\
\text { psychosocial circumstances }\end{array}$ & 127694 & 0.0753 & 0.0401 & 1.8759 & 1.7653 & 1.9935 \\
\hline K25 & K25.9 & $\begin{array}{l}\text { Gastric ulcer, unsp as acute or } \\
\text { chronic, w/o hemor or perf }\end{array}$ & 5442 & 0.0985 & 0.0526 & 1.8727 & 1.5718 & 2.2312 \\
\hline K86 & K86.1 & Other chronic pancreatitis & 5781 & 0.0983 & 0.0526 & 1.8696 & 1.5768 & 2.2168 \\
\hline K68 & K68.11 & $\begin{array}{l}\text { Postprocedural retroperitoneal } \\
\text { abscess }\end{array}$ & 4165 & 0.0984 & 0.0528 & 1.8654 & 1.5280 & 2.2774 \\
\hline $\mathrm{H} 18$ & $\mathrm{H} 18.51$ & Endothelial corneal dystrophy & 11506 & 0.0961 & 0.0518 & 1.8546 & 1.6380 & 2.0999 \\
\hline T81 & $\begin{array}{c}\text { T81.89X } \\
\mathrm{A}\end{array}$ & $\begin{array}{l}\text { Oth complications of procedures, } \\
\text { NEC, init }\end{array}$ & 10309 & 0.0957 & 0.0520 & 1.8411 & 1.6150 & 2.0989 \\
\hline D51 & D51.9 & $\begin{array}{l}\text { Vitamin B12 deficiency anemia, } \\
\text { unspecified }\end{array}$ & 14069 & 0.0948 & 0.0515 & 1.8398 & 1.6416 & 2.0619 \\
\hline D29 & D29.1 & Benign neoplasm of prostate & 6819 & 0.0963 & 0.0524 & 1.8370 & 1.5670 & 2.1535 \\
\hline Z44 & Z44.8 & $\begin{array}{l}\text { Encounter for fit/adjst of external } \\
\text { prosthetic devices }\end{array}$ & 5211 & 0.0967 & 0.0527 & 1.8369 & 1.5333 & 2.2005 \\
\hline M84 & $\begin{array}{l}\text { M84.369 } \\
\mathrm{S}\end{array}$ & $\begin{array}{l}\text { Stress fracture, unspecified tibia } \\
\text { and fibula, sequela }\end{array}$ & 5832 & 0.0965 & 0.0526 & 1.8362 & 1.5472 & 2.1791 \\
\hline $\mathrm{C} 43$ & C43.9 & $\begin{array}{l}\text { Malignant melanoma of skin, } \\
\text { unspecified }\end{array}$ & 4224 & 0.0968 & 0.0528 & 1.8345 & 1.5020 & 2.2405 \\
\hline R82 & R82.5 & $\begin{array}{l}\text { Elevated urine levels of } \\
\text { drug/meds/biol subst }\end{array}$ & 14186 & 0.0945 & 0.0515 & 1.8328 & 1.6357 & 2.0537 \\
\hline R35 & R35.0 & Frequency of micturition & 39834 & 0.0890 & 0.0486 & 1.8321 & 1.6989 & 1.9756 \\
\hline L82 & L82.1 & Other seborrheic keratosis & 55603 & 0.0859 & 0.0470 & 1.8281 & 1.7077 & 1.9571 \\
\hline Z91 & Z91.19 & $\begin{array}{l}\text { Patient's noncompliance w oth } \\
\text { medical treatment and regimen }\end{array}$ & 64332 & 0.0842 & 0.0461 & 1.8244 & 1.7083 & 1.9482 \\
\hline R49 & R49.0 & Dysphonia & 12308 & 0.0942 & 0.0518 & 1.8197 & 1.6114 & 2.0550 \\
\hline G62 & G62.9 & Polyneuropathy, unspecified & 14108 & 0.0937 & 0.0516 & 1.8167 & 1.6201 & 2.0371 \\
\hline $\mathrm{H} 43$ & H43.819 & $\begin{array}{l}\text { Vitreous degeneration, } \\
\text { unspecified eye }\end{array}$ & 38102 & 0.0887 & 0.0489 & 1.8155 & 1.6812 & 1.9606 \\
\hline 183 & 183.90 & $\begin{array}{l}\text { Asymptomatic varicose veins of } \\
\text { unspecified lower extremity }\end{array}$ & 14263 & 0.0934 & 0.0516 & 1.8107 & 1.6154 & 2.0297 \\
\hline S22 & $\begin{array}{l}\text { S22.39X } \\
\text { A }\end{array}$ & $\begin{array}{l}\text { Fracture of one rib, unsp side, } \\
\text { init for clos } \mathrm{fx}\end{array}$ & 10436 & 0.0942 & 0.0520 & 1.8102 & 1.5873 & 2.0644 \\
\hline S31 & $\begin{array}{l}\text { S31.000 } \\
\mathrm{A}\end{array}$ & $\begin{array}{l}\text { Unsp opn wnd low back and pelv } \\
\text { w/o penet retroperiton, init }\end{array}$ & 10430 & 0.0942 & 0.0520 & 1.8093 & 1.5864 & 2.0635 \\
\hline $\mathrm{Z13}$ & Z13.6 & $\begin{array}{l}\text { Encounter for screening for } \\
\text { cardiovascular disorders }\end{array}$ & 237211 & 0.0616 & 0.0341 & 1.8054 & 1.6707 & 1.9509 \\
\hline K57 & K57.30 & $\begin{array}{l}\text { Dvrtclos of Ig int w/o perforation } \\
\text { or abscess w/o bleeding }\end{array}$ & 60553 & 0.0842 & 0.0466 & 1.8047 & 1.6880 & 1.9295 \\
\hline R06 & R06.02 & Shortness of breath & 118999 & 0.0750 & 0.0417 & 1.7984 & 1.6926 & 1.9108 \\
\hline R09 & R09.81 & Nasal congestion & 40481 & 0.0871 & 0.0488 & 1.7869 & 1.6567 & 1.9274 \\
\hline H31 & H31.009 & $\begin{array}{l}\text { Unspecified chorioretinal scars, } \\
\text { unspecified eye }\end{array}$ & 9734 & 0.0931 & 0.0522 & 1.7845 & 1.5566 & 2.0458 \\
\hline$\overline{Z 23}$ & Z23. & Encounter for immunization & 260232 & 0.0594 & 0.0336 & 1.7694 & 1.6229 & 1.9292 \\
\hline $\mathrm{D} 12$ & D12.0 & Benign neoplasm of cecum & 65120 & 0.0821 & 0.0465 & 1.7646 & 1.6519 & 1.8850 \\
\hline K92 & K92.1 & Melena & 40170 & 0.0860 & 0.0489 & 1.7578 & 1.6287 & 1.8971 \\
\hline S01 & $\begin{array}{l}\text { S01.00X } \\
\mathrm{A}\end{array}$ & $\begin{array}{l}\text { Unspecified open wound of } \\
\text { scalp, initial encounter }\end{array}$ & 7728 & 0.0921 & 0.0524 & 1.7574 & 1.5076 & 2.0485 \\
\hline L21 & L21.9 & $\begin{array}{l}\text { Seborrheic dermatitis, } \\
\text { unspecified }\end{array}$ & 25335 & 0.0885 & 0.0505 & 1.7534 & 1.6012 & 1.9200 \\
\hline
\end{tabular}


medRxiv preprint doi: https://doi.org/10.1101/2022.01.22.22269694; this version posted February 21, 2022. The copyright holder for this preprint (which was not certified by peer review) is the author/funder, who has granted medRxiv a license to display the preprint in perpetuity. This article is a US Government work. It is not subject to copyright under 17 USC 105 and is also made available for use under a CCO license.

\begin{tabular}{|c|c|c|c|c|c|c|c|c|}
\hline R79 & R79.89 & $\begin{array}{l}\text { Other specified abnormal } \\
\text { findings of blood chemistry }\end{array}$ & 28403 & 0.0879 & 0.0502 & 1.7521 & 1.6065 & 1.9110 \\
\hline K80 & K80.20 & $\begin{array}{l}\text { Calculus of gallbladder w/o } \\
\text { cholecystitis w/o obstruction }\end{array}$ & 16844 & 0.0896 & 0.0514 & 1.7429 & 1.5645 & 1.9417 \\
\hline R31 & R31.9 & Hematuria, unspecified & 42562 & 0.0844 & 0.0489 & 1.7277 & 1.6025 & 1.8627 \\
\hline L03 & L03.90 & Cellulitis, unspecified & 70833 & 0.0800 & 0.0463 & 1.7269 & 1.6183 & 1.8429 \\
\hline R42 & R42. & Dizziness and giddiness & 67634 & 0.0800 & 0.0467 & 1.7131 & 1.6040 & 1.8297 \\
\hline D48 & D48.5 & $\begin{array}{l}\text { Neoplasm of uncertain behavior } \\
\text { of skin }\end{array}$ & 44749 & 0.0834 & 0.0488 & 1.7106 & 1.5883 & 1.8424 \\
\hline Z98 & Z98.890 & $\begin{array}{l}\text { Other specified postprocedural } \\
\text { states }\end{array}$ & 92911 & 0.0763 & 0.0447 & 1.7083 & 1.6057 & 1.8174 \\
\hline R39 & R39.15 & Urgency of urination & 29413 & 0.0858 & 0.0503 & 1.7069 & 1.5654 & 1.8611 \\
\hline K31 & K31.89 & $\begin{array}{l}\text { Other diseases of stomach and } \\
\text { duodenum }\end{array}$ & 15241 & 0.0872 & 0.0517 & 1.6854 & 1.5028 & 1.8900 \\
\hline M89 & M89.9 & Disorder of bone, unspecified & 17841 & 0.0863 & 0.0515 & 1.6760 & 1.5056 & 1.8657 \\
\hline $\mathrm{R} 25$ & R25.2 & Cramp and spasm & 28206 & 0.0846 & 0.0505 & 1.6743 & 1.5323 & 1.8294 \\
\hline R69 & R69. & Illness, unspecified & 161563 & 0.0675 & 0.0405 & 1.6691 & 1.5685 & 1.7761 \\
\hline Z09 & Z09. & $\begin{array}{l}\text { Encntr for f/u exam aft trtmt for } \\
\text { cond oth than malig neoplm }\end{array}$ & 55214 & 0.0803 & 0.0481 & 1.6682 & 1.5555 & 1.7891 \\
\hline Z48 & Z48.02 & Encounter for removal of sutures & 78659 & 0.0770 & 0.0462 & 1.6660 & 1.5627 & 1.7760 \\
\hline M20 & M20.40 & $\begin{array}{l}\text { Other hammer toe(s) (acquired), } \\
\text { unspecified foot }\end{array}$ & 43692 & 0.0811 & 0.0492 & 1.6482 & 1.5280 & 1.7779 \\
\hline L98 & L98.9 & $\begin{array}{l}\text { Disorder of the skin and } \\
\text { subcutaneous tissue, unspecified }\end{array}$ & 55257 & 0.0784 & 0.0485 & 1.6182 & 1.5080 & 1.7365 \\
\hline M19 & M19.90 & $\begin{array}{l}\text { Unspecified osteoarthritis, } \\
\text { unspecified site }\end{array}$ & 89625 & 0.0737 & 0.0460 & 1.6021 & 1.5046 & 1.7059 \\
\hline F12 & F12.10 & Cannabis abuse, uncomplicated & 24113 & 0.0346 & 0.0548 & 0.6324 & 0.5463 & 0.7320 \\
\hline K58 & K58.9 & $\begin{array}{l}\text { Irritable bowel syndrome without } \\
\text { diarrhea }\end{array}$ & 17352 & 0.0329 & 0.0544 & 0.6046 & 0.5071 & 0.7207 \\
\hline R51 & R51. & Headache & 51370 & 0.0335 & 0.0569 & 0.5896 & 0.5310 & 0.6546 \\
\hline L70 & L70.0 & Acne vulgaris & 12335 & 0.0317 & 0.0541 & 0.5854 & 0.4739 & 0.7233 \\
\hline M22 & M22.40 & $\begin{array}{l}\text { Chondromalacia patellae, } \\
\text { unspecified knee }\end{array}$ & 16168 & 0.0317 & 0.0544 & 0.5820 & 0.4835 & 0.7005 \\
\hline F15 & F15.20 & $\begin{array}{l}\text { Other stimulant dependence, } \\
\text { uncomplicated }\end{array}$ & 8910 & 0.0300 & 0.0540 & 0.5554 & 0.4302 & 0.7168 \\
\hline Z56 & $Z 56.0$ & Unemployment, unspecified & 34996 & 0.0298 & 0.0560 & 0.5319 & 0.4661 & 0.6069 \\
\hline A63 & A63.0 & Anogenital (venereal) warts & 4871 & 0.0279 & 0.0537 & 0.5199 & 0.3639 & 0.7428 \\
\hline T74 & $\begin{array}{c}\mathrm{T74.21X} \\
\mathrm{A}\end{array}$ & $\begin{array}{l}\text { Adult sexual abuse, confirmed, } \\
\text { initial encounter }\end{array}$ & 3385 & 0.0278 & 0.0536 & 0.5182 & 0.3376 & 0.7955 \\
\hline Z20 & Z20.828 & $\begin{array}{l}\text { Contact } w \text { and exposure to oth } \\
\text { viral communicable diseases }\end{array}$ & 19317 & 0.0279 & 0.0549 & 0.5086 & 0.4243 & 0.6096 \\
\hline $\mathrm{X} 50$ & $\begin{array}{c}\mathrm{X} 50.0 \mathrm{XX} \\
\mathrm{A}\end{array}$ & $\begin{array}{l}\text { Overexertion from strenuous } \\
\text { movement or load, init }\end{array}$ & 3150 & 0.0244 & 0.0536 & 0.4561 & 0.2838 & 0.7327 \\
\hline $\mathrm{J} 03$ & $\mathrm{~J} 03.90$ & Acute tonsillitis, unspecified & 5855 & 0.0243 & 0.0538 & 0.4505 & 0.3175 & 0.6391 \\
\hline A60 & A60.9 & $\begin{array}{l}\text { Anogenital herpesviral infection, } \\
\text { unspecified }\end{array}$ & 7165 & 0.0241 & 0.0540 & 0.4475 & 0.3258 & 0.6145 \\
\hline Z31 & Z31.5 & $\begin{array}{l}\text { Encounter for procreative genetic } \\
\text { counseling }\end{array}$ & 2984 & 0.0221 & 0.0536 & 0.4126 & 0.2471 & 0.6889 \\
\hline G43 & G43.909 & $\begin{array}{l}\text { Migraine, unsp, not intractable, } \\
\text { without status migrainosus }\end{array}$ & 42103 & 0.0231 & 0.0576 & 0.4016 & 0.3503 & 0.4605 \\
\hline N95 & N95.1 & $\begin{array}{l}\text { Menopausal and female } \\
\text { climacteric states }\end{array}$ & 9036 & 0.0216 & 0.0542 & 0.3982 & 0.2952 & 0.5371 \\
\hline R92 & R92.8 & $\begin{array}{l}\text { Oth abn and inconclusive } \\
\text { findings on } \mathrm{dx} \text { imaging of breast }\end{array}$ & 3749 & 0.0195 & 0.0537 & 0.3626 & 0.2225 & 0.5907 \\
\hline N60 & N60.19 & $\begin{array}{l}\text { Diffuse cystic mastopathy of } \\
\text { unspecified breast }\end{array}$ & 3693 & 0.0187 & 0.0537 & 0.3479 & 0.2105 & 0.5748 \\
\hline A64 & A64. & $\begin{array}{l}\text { Unspecified sexually transmitted } \\
\text { disease }\end{array}$ & 6612 & 0.0180 & 0.0540 & 0.3331 & 0.2271 & 0.4886 \\
\hline N85 & N85.8 & $\begin{array}{l}\text { Other specified noninflammatory } \\
\text { disorders of uterus }\end{array}$ & 3702 & 0.0170 & 0.0537 & 0.3167 & 0.1872 & 0.5360 \\
\hline
\end{tabular}


medRxiv preprint doi: https://doi.org/10.1101/2022.01.22.22269694; this version posted February 21, 2022. The copyright holder for this preprint (which was not certified by peer review) is the author/funder, who has granted medRxiv a license to display the preprint in perpetuity.

This article is a US Government work. It is not subject to copyright under 17 USC 105 and is also made available for use under a CCO license.

COMORBIDITIES IN MODELS FOR COVID-19 DEATH

\begin{tabular}{|c|c|c|c|c|c|c|c|c|}
\hline N46 & N46.9 & Male infertility, unspecified & 2364 & 0.0161 & 0.0536 & 0.2999 & 0.1524 & 0.5904 \\
\hline N84 & N84.1 & Polyp of cervix uteri & 1403 & 0.0157 & 0.0535 & 0.2932 & 0.1204 & 0.7138 \\
\hline N73 & N73.9 & $\begin{array}{l}\text { Female pelvic inflammatory } \\
\text { disease, unspecified }\end{array}$ & 1373 & 0.0138 & 0.0535 & 0.2587 & 0.0992 & 0.6745 \\
\hline L68 & L68.0 & Hirsutism & 1024 & 0.0127 & 0.0535 & 0.2375 & 0.0745 & 0.7569 \\
\hline N83 & N83.20 & Unspecified ovarian cysts & 5025 & 0.0127 & 0.0539 & 0.2361 & 0.1400 & 0.3984 \\
\hline N89 & N89.8 & $\begin{array}{l}\text { Other specified noninflammatory } \\
\text { disorders of vagina }\end{array}$ & 4793 & 0.0123 & 0.0539 & 0.2283 & 0.1324 & 0.3937 \\
\hline D25 & D25.9 & $\begin{array}{l}\text { Leiomyoma of uterus, } \\
\text { unspecified }\end{array}$ & 4634 & 0.0123 & 0.0539 & 0.2282 & 0.1311 & 0.3972 \\
\hline N90 & N90.7 & Vulvar cyst & 988 & 0.0121 & 0.0534 & 0.2272 & 0.0680 & 0.7594 \\
\hline F90 & F90.9 & $\begin{array}{l}\text { Attention-deficit hyperactivity } \\
\text { disorder, unspecified type }\end{array}$ & 11738 & 0.0121 & 0.0548 & 0.2207 & 0.1552 & 0.3139 \\
\hline A74 & A74.9 & Chlamydial infection, unspecified & 1082 & 0.0102 & 0.0535 & 0.1901 & 0.0539 & 0.6713 \\
\hline N76 & N76.0 & Acute vaginitis & 10023 & 0.0098 & 0.0547 & 0.1789 & 0.1171 & 0.2733 \\
\hline E28 & E28.2 & Polycystic ovarian syndrome & 2511 & 0.0088 & 0.0537 & 0.1633 & 0.0669 & 0.3987 \\
\hline N94 & N94.6 & Dysmenorrhea, unspecified & 9013 & 0.0081 & 0.0546 & 0.1484 & 0.0908 & 0.2425 \\
\hline N92 & N92.0 & $\begin{array}{l}\text { Excessive and frequent } \\
\text { menstruation with regular cycle }\end{array}$ & 9748 & 0.0081 & 0.0547 & 0.1483 & 0.0925 & 0.2377 \\
\hline N77 & N77.1 & $\begin{array}{l}\text { Vaginitis, vulvitis and } \\
\text { vulvovaginitis in dis classd } \\
\text { elswhr }\end{array}$ & 1805 & 0.0078 & 0.0536 & 0.1448 & 0.0473 & 0.4436 \\
\hline N72 & N72. & $\begin{array}{l}\text { Inflammatory disease of cervix } \\
\text { uteri }\end{array}$ & 1687 & 0.0071 & 0.0536 & 0.1328 & 0.0396 & 0.4452 \\
\hline R87 & R87.619 & $\begin{array}{l}\text { Unsp abnormal cytolog findings } \\
\text { in specmn from cervix uteri }\end{array}$ & 6501 & 0.0071 & 0.0542 & 0.1305 & 0.0703 & 0.2422 \\
\hline N80 & N80.9 & Endometriosis, unspecified & 2081 & 0.0067 & 0.0536 & 0.1255 & 0.0409 & 0.3846 \\
\hline N87 & N87.9 & $\begin{array}{l}\text { Dysplasia of cervix uteri, } \\
\text { unspecified }\end{array}$ & 2020 & 0.0064 & 0.0536 & 0.1200 & 0.0375 & 0.3839 \\
\hline A56 & A56.00 & $\begin{array}{l}\text { Chlamydial infection of lower } \\
\text { genitourinary tract, unsp }\end{array}$ & 1100 & 0.0064 & 0.0535 & 0.1190 & 0.0244 & 0.5801 \\
\hline N97 & N97.9 & Female infertility, unspecified & 1964 & 0.0051 & 0.0536 & 0.0950 & 0.0252 & 0.3578 \\
\hline Z30 & Z30.09 & $\begin{array}{l}\text { Encounter for oth general cnsl } \\
\text { and advice on contraception }\end{array}$ & 15303 & 0.0050 & 0.0556 & 0.0893 & 0.0551 & 0.1446 \\
\hline N91 & N91.2 & Amenorrhea, unspecified & 2649 & 0.0042 & 0.0537 & 0.0773 & 0.0218 & 0.2740 \\
\hline N93 & N93.9 & $\begin{array}{l}\text { Abnormal uterine and vaginal } \\
\text { bleeding, unspecified }\end{array}$ & 5282 & 0.0042 & 0.0541 & 0.0770 & 0.0315 & 0.1884 \\
\hline Z32 & Z32.02 & $\begin{array}{l}\text { Encounter for pregnancy test, } \\
\text { result negative }\end{array}$ & 6339 & 0.0024 & 0.0543 & 0.0436 & 0.0147 & 0.1289 \\
\hline Z33 & Z33.1 & Pregnant state, incidental & 3827 & 0.0010 & 0.0539 & 0.0194 & 0.0024 & 0.1585 \\
\hline
\end{tabular}


TABLE 2: MULTIVARIATE MODEL FOR COVID-19 DEATH BASED UPON PRE-EXISTING CONDITIONS

\section{*Most Common ICD-10 Code and Description for Each Category Diagnosis ${ }^{*}$ Ranked in Descending Order by Contribution to the Logit Function}

\begin{tabular}{|c|c|c|c|c|c|c|c|c|c|c|}
\hline $\begin{array}{l}\text { RootICD- } \\
10\end{array}$ & $\begin{array}{l}\text { ICD-10 } \\
\text { Code* }^{*}\end{array}$ & ICD-10 Description* & Cases & Coefficient & Std Error & Z_Score & P_Value & $\begin{array}{l}\text { Lower } \\
\mathrm{Cl}\end{array}$ & $\begin{array}{l}\text { Higher } \\
\text { Cl }\end{array}$ & Coeff_RR ${ }^{\star \star}$ \\
\hline 110 & 110. & Essential (primary) hypertension & 208817 & 0.1934 & 0.0075 & 25.8300 & 0.0000 & 0.1787 & 0.2081 & 0.7424 \\
\hline J64 & J64. & Unspecified pneumoconiosis & 107 & 0.2495 & 0.1007 & 2.4800 & 0.0130 & 0.0521 & 0.4468 & 0.6695 \\
\hline F03 & F03.90 & $\begin{array}{l}\text { Unspecified dementia without behavioral } \\
\text { disturbance }\end{array}$ & 13895 & 0.1619 & 0.0076 & 21.3500 & 0.0000 & 0.1471 & 0.1768 & 0.6683 \\
\hline C93 & C93.10 & $\begin{array}{l}\text { Chronic myelomonocytic leukemia not } \\
\text { achieve remission }\end{array}$ & 122 & 0.2194 & 0.0815 & 2.6900 & 0.0070 & 0.0597 & 0.3791 & 0.6583 \\
\hline $\mathrm{C} 24$ & C24.1 & Malignant neoplasm of ampulla of Vater & 122 & 0.2489 & 0.1006 & 2.4700 & 0.0130 & 0.0517 & 0.4461 & 0.6318 \\
\hline $\mathrm{C} 71$ & C71.9 & Malignant neoplasm of brain, unspecified & 557 & 0.3400 & 0.0786 & 4.3300 & 0.0000 & 0.1860 & 0.4941 & 0.5782 \\
\hline C91 & C91.10 & $\begin{array}{l}\text { Chronic lymphocytic leuk of B-cell type not } \\
\text { achieve remis }\end{array}$ & 1854 & 0.2492 & 0.0356 & 7.0000 & 0.0000 & 0.1794 & 0.3189 & 0.5362 \\
\hline G70 & G70.00 & $\begin{array}{l}\text { Myasthenia gravis without (acute) } \\
\text { exacerbation }\end{array}$ & 745 & 0.2897 & 0.0625 & 4.6300 & 0.0000 & 0.1671 & 0.4122 & 0.5229 \\
\hline D46 & D46.9 & Myelodysplastic syndrome, unspecified & 755 & 0.1633 & 0.0333 & 4.9000 & 0.0000 & 0.0980 & 0.2286 & 0.4858 \\
\hline L12 & L12.0 & Bullous pemphigoid & 334 & 0.1955 & 0.0703 & 2.7800 & 0.0050 & 0.0577 & 0.3334 & 0.4425 \\
\hline C95 & C95.90 & $\begin{array}{l}\text { Leukemia, unspecified not having achieved } \\
\text { remission }\end{array}$ & 609 & 0.1530 & 0.0476 & 3.2200 & 0.0010 & 0.0598 & 0.2463 & 0.4055 \\
\hline N18 & N18.9 & Chronic kidney disease, unspecified & 48428 & 0.1663 & 0.0105 & 15.8500 & 0.0000 & 0.1457 & 0.1868 & 0.3998 \\
\hline G20 & G20. & Parkinson's disease & 5105 & 0.1656 & 0.0196 & 8.4500 & 0.0000 & 0.1272 & 0.2040 & 0.3912 \\
\hline C78 & C78.7 & $\begin{array}{l}\text { Secondary malig neoplasm of liver and } \\
\text { intrahepatic bile duct }\end{array}$ & 1305 & 0.1545 & 0.0345 & 4.4700 & 0.0000 & 0.0868 & 0.2222 & 0.3834 \\
\hline G30 & G30.9 & Alzheimer's disease, unspecified & 4348 & 0.0866 & 0.0107 & 8.0700 & 0.0000 & 0.0655 & 0.1076 & 0.3712 \\
\hline J84 & J84.10 & Pulmonary fibrosis, unspecified & 7398 & 0.2074 & 0.0225 & 9.2100 & 0.0000 & 0.1633 & 0.2516 & 0.3634 \\
\hline $\mathrm{C} 85$ & C85.80 & $\begin{array}{l}\text { Oth types of non-Hodgkin lymphoma, } \\
\text { unspecified site }\end{array}$ & 2228 & 0.2301 & 0.0451 & 5.1000 & 0.0000 & 0.1417 & 0.3185 & 0.3588 \\
\hline R18 & R18.8 & Other ascites & 2408 & 0.1669 & 0.0335 & 4.9800 & 0.0000 & 0.1013 & 0.2325 & 0.3367 \\
\hline G11 & G11.1 & Early-onset cerebellar ataxia & 586 & 0.1685 & 0.0640 & 2.6300 & 0.0080 & 0.0431 & 0.2939 & 0.3292 \\
\hline C34 & C34.90 & $\begin{array}{l}\text { Malignant neoplasm of unsp part of unsp } \\
\text { bronchus or lung }\end{array}$ & 3805 & 0.1404 & 0.0272 & 5.1500 & 0.0000 & 0.0870 & 0.1938 & 0.3083 \\
\hline Z66 & Z66. & Do not resuscitate & 6448 & 0.0869 & 0.0105 & 8.2600 & 0.0000 & 0.0663 & 0.1075 & 0.3030 \\
\hline F02 & F02.80 & $\begin{array}{l}\text { Dementia in oth diseases classd elswhr } \\
\text { w/o behavrl disturb }\end{array}$ & 8684 & 0.0775 & 0.0101 & 7.7100 & 0.0000 & 0.0578 & 0.0972 & 0.3028 \\
\hline
\end{tabular}




\begin{tabular}{|c|c|c|c|c|c|c|c|c|c|c|}
\hline R26 & R26.9 & $\begin{array}{l}\text { Unspecified abnormalities of gait and } \\
\text { mobility }\end{array}$ & 67067 & 0.1327 & 0.0091 & 14.5100 & 0.0000 & 0.1148 & 0.1506 & 0.2996 \\
\hline C79 & C79.51 & Secondary malignant neoplasm of bone & 2724 & 0.1445 & 0.0299 & 4.8300 & 0.0000 & 0.0859 & 0.2031 & 0.2994 \\
\hline G12 & G12.21 & Amyotrophic lateral sclerosis & 685 & 0.2008 & 0.0842 & 2.3900 & 0.0170 & 0.0359 & 0.3658 & 0.2954 \\
\hline $\mathrm{C} 22$ & $\mathrm{C} 22.0$ & Liver cell carcinoma & 1150 & 0.1762 & 0.0596 & 2.9600 & 0.0030 & 0.0594 & 0.2929 & 0.2889 \\
\hline $\mathrm{D} 02$ & D02.20 & $\begin{array}{l}\text { Carcinoma in situ of unspecified bronchus } \\
\text { and lung }\end{array}$ & 2023 & 0.1246 & 0.0342 & 3.6500 & 0.0000 & 0.0577 & 0.1915 & 0.2875 \\
\hline C18 & C18.9 & Malignant neoplasm of colon, unspecified & 4296 & 0.1874 & 0.0333 & 5.6300 & 0.0000 & 0.1221 & 0.2526 & 0.2845 \\
\hline R64 & R64. & Cachexia & 797 & 0.1066 & 0.0384 & 2.7800 & 0.0060 & 0.0313 & 0.1818 & 0.2820 \\
\hline N40 & N40.0 & $\begin{array}{l}\text { Benign prostatic hyperplasia without lower } \\
\text { urinry tract symp }\end{array}$ & 85767 & 0.1412 & 0.0103 & 13.6600 & 0.0000 & 0.1209 & 0.1615 & 0.2615 \\
\hline C90 & C90.00 & $\begin{array}{l}\text { Multiple myeloma not having achieved } \\
\text { remission }\end{array}$ & 1041 & 0.1577 & 0.0595 & 2.6500 & 0.0080 & 0.0411 & 0.2743 & 0.2591 \\
\hline 125 & 125.10 & $\begin{array}{l}\text { Athscl heart disease of native coronary } \\
\text { artery w/o ang pctrs }\end{array}$ & 77259 & 0.1158 & 0.0095 & 12.1900 & 0.0000 & 0.0972 & 0.1344 & 0.2557 \\
\hline Z96 & Z96.1 & Presence of intraocular lens & 58557 & 0.1228 & 0.0101 & 12.2200 & 0.0000 & 0.1031 & 0.1425 & 0.2537 \\
\hline E11 & E11.9 & $\begin{array}{l}\text { Type } 2 \text { diabetes mellitus without } \\
\text { complications }\end{array}$ & 114394 & 0.1488 & 0.0118 & 12.5700 & 0.0000 & 0.1256 & 0.1720 & 0.2393 \\
\hline Z74 & Z74.09 & Other reduced mobility & 17940 & 0.0917 & 0.0102 & 8.9500 & 0.0000 & 0.0716 & 0.1117 & 0.2368 \\
\hline L89 & L89.90 & $\begin{array}{l}\text { Pressure ulcer of unspecified site, } \\
\text { unspecified stage }\end{array}$ & 8092 & 0.0834 & 0.0130 & 6.4000 & 0.0000 & 0.0579 & 0.1090 & 0.2350 \\
\hline C32 & C32.9 & Malignant neoplasm of larynx, unspecified & 930 & 0.1421 & 0.0653 & 2.1700 & 0.0300 & 0.0140 & 0.2701 & 0.2291 \\
\hline J61 & J61. & $\begin{array}{l}\text { Pneumoconiosis due to asbestos and } \\
\text { other mineral fibers }\end{array}$ & 1189 & 0.1202 & 0.0467 & 2.5700 & 0.0100 & 0.0287 & 0.2116 & 0.2271 \\
\hline R54 & R54. & Age-related physical debility & 11357 & 0.0696 & 0.0096 & 7.2600 & 0.0000 & 0.0508 & 0.0884 & 0.2160 \\
\hline J44 & $\mathrm{J} 44.9$ & $\begin{array}{l}\text { Chronic obstructive pulmonary disease, } \\
\text { unspecified }\end{array}$ & 63653 & 0.1281 & 0.0121 & 10.5500 & 0.0000 & 0.1043 & 0.1519 & 0.2160 \\
\hline 148 & 148.91 & Unspecified atrial fibrillation & 35951 & 0.1048 & 0.0114 & 9.1600 & 0.0000 & 0.0824 & 0.1272 & 0.2035 \\
\hline 196 & 196. & Gangrene, not elsewhere classified & 2079 & 0.0857 & 0.0322 & 2.6600 & 0.0080 & 0.0225 & 0.1488 & 0.1925 \\
\hline 137 & 137.0 & Nonrheumatic pulmonary valve stenosis & 1552 & 0.1488 & 0.0670 & 2.2200 & 0.0260 & 0.0175 & 0.2800 & 0.1912 \\
\hline E78 & E78.5 & Hyperlipidemia, unspecified & 223873 & 0.0921 & 0.0127 & 7.2500 & 0.0000 & 0.0672 & 0.1170 & 0.1897 \\
\hline 171 & 171.4 & $\begin{array}{l}\text { Abdominal aortic aneurysm, without } \\
\text { rupture }\end{array}$ & 12513 & 0.1183 & 0.0192 & 6.1800 & 0.0000 & 0.0808 & 0.1559 & 0.1896 \\
\hline 150 & 150.9 & Heart failure, unspecified & 36427 & 0.0771 & 0.0102 & 7.5800 & 0.0000 & 0.0572 & 0.0971 & 0.1863 \\
\hline $\mathrm{H} 25$ & $\mathrm{H} 25.13$ & Age-related nuclear cataract, bilateral & 127537 & 0.1014 & 0.0129 & 7.8700 & 0.0000 & 0.0762 & 0.1267 & 0.1799 \\
\hline 185 & 185.00 & Esophageal varices without bleeding & 2480 & 0.1587 & 0.0712 & 2.2300 & 0.0260 & 0.0192 & 0.2983 & 0.1764 \\
\hline J96 & J96.01 & Acute respiratory failure with hypoxia & 13350 & 0.0753 & 0.0135 & 5.5600 & 0.0000 & 0.0488 & 0.1019 & 0.1749 \\
\hline N19 & N19. & Unspecified kidney failure & 7383 & 0.0749 & 0.0170 & 4.4200 & 0.0000 & 0.0417 & 0.1082 & 0.1702 \\
\hline J47 & J47.9 & Bronchiectasis, uncomplicated & 1749 & 0.0978 & 0.0454 & 2.1600 & 0.0310 & 0.0089 & 0.1867 & 0.1656 \\
\hline M81 & M81.0 & $\begin{array}{l}\text { Age-related osteoporosis w/o current } \\
\text { pathological fracture }\end{array}$ & 10016 & 0.1163 & 0.0249 & 4.6700 & 0.0000 & 0.0675 & 0.1652 & 0.1645 \\
\hline D04 & D04.9 & Carcinoma in situ of skin, unspecified & 3845 & 0.1033 & 0.0345 & 3.0000 & 0.0030 & 0.0357 & 0.1708 & 0.1639 \\
\hline $\mathrm{H} 27$ & $\mathrm{H} 27.8$ & Other specified disorders of lens & 6825 & 0.0739 & 0.0173 & 4.2800 & 0.0000 & 0.0401 & 0.1078 & 0.1638 \\
\hline
\end{tabular}




\begin{tabular}{|c|c|c|c|c|c|c|c|c|c|c|}
\hline $\mathrm{H} 90$ & H90.3 & Sensorineural hearing loss, bilateral & 118138 & 0.1200 & 0.0145 & 8.2600 & 0.0000 & 0.0915 & 0.1485 & 0.1634 \\
\hline E03 & E03.9 & Hypothyroidism, unspecified & 35891 & 0.1812 & 0.0244 & 7.4300 & 0.0000 & 0.1334 & 0.2290 & 0.1631 \\
\hline D64 & D64.9 & Anemia, unspecified & 62258 & 0.0916 & 0.0122 & 7.5300 & 0.0000 & 0.0677 & 0.1154 & 0.1576 \\
\hline B02 & B02.9 & Zoster without complications & 16479 & 0.1667 & 0.0329 & 5.0700 & 0.0000 & 0.1022 & 0.2312 & 0.1520 \\
\hline N25 & N25.81 & $\begin{array}{l}\text { Secondary hyperparathyroidism of renal } \\
\text { origin }\end{array}$ & 6475 & 0.0635 & 0.0191 & 3.3300 & 0.0010 & 0.0261 & 0.1008 & 0.1478 \\
\hline 173 & 173.9 & Peripheral vascular disease, unspecified & 31350 & 0.0715 & 0.0114 & 6.2800 & 0.0000 & 0.0492 & 0.0938 & 0.1469 \\
\hline $\mathrm{H} 61$ & $\mathrm{H} 61.23$ & Impacted cerumen, bilateral & 58221 & 0.1234 & 0.0162 & 7.6000 & 0.0000 & 0.0916 & 0.1552 & 0.1453 \\
\hline Z43 & Z43.3 & Encounter for attention to colostomy & 2992 & 0.0951 & 0.0412 & 2.3100 & 0.0210 & 0.0144 & 0.1758 & 0.1428 \\
\hline H91 & $\mathrm{H} 91.90$ & Unspecified hearing loss, unspecified ear & 71156 & 0.1438 & 0.0204 & 7.0600 & 0.0000 & 0.1039 & 0.1838 & 0.1408 \\
\hline $\mathrm{H} 26$ & $\mathrm{H} 26.9$ & Unspecified cataract & 60786 & 0.0719 & 0.0110 & 6.5600 & 0.0000 & 0.0504 & 0.0933 & 0.1370 \\
\hline H35 & H35.31 & $\begin{array}{l}\text { Nonexudative age-related macular } \\
\text { degeneration }\end{array}$ & 60393 & 0.0900 & 0.0132 & 6.8200 & 0.0000 & 0.0641 & 0.1158 & 0.1344 \\
\hline C67 & C67.9 & $\begin{array}{l}\text { Malignant neoplasm of bladder, } \\
\text { unspecified }\end{array}$ & 4135 & 0.0867 & 0.0338 & 2.5600 & 0.0100 & 0.0203 & 0.1530 & 0.1324 \\
\hline Z95 & Z95.1 & Presence of aortocoronary bypass graft & 34407 & 0.0659 & 0.0121 & 5.4600 & 0.0000 & 0.0422 & 0.0896 & 0.1317 \\
\hline R62 & $\mathrm{R} 62.7$ & Adult failure to thrive & 3414 & 0.0395 & 0.0150 & 2.6400 & 0.0080 & 0.0102 & 0.0689 & 0.1295 \\
\hline J90 & J90. & Pleural effusion, not elsewhere classified & 5673 & 0.0519 & 0.0175 & 2.9600 & 0.0030 & 0.0176 & 0.0862 & 0.1257 \\
\hline R63 & R63.4 & Abnormal weight loss & 34666 & 0.1042 & 0.0190 & 5.4700 & 0.0000 & 0.0669 & 0.1415 & 0.1253 \\
\hline J43 & J43.9 & Emphysema, unspecified & 9950 & 0.0875 & 0.0256 & 3.4100 & 0.0010 & 0.0372 & 0.1377 & 0.1241 \\
\hline L97 & L97.509 & $\begin{array}{l}\text { Non-pressure chronic ulcer oth prt unsp } \\
\text { foot w unsp severity }\end{array}$ & 13950 & 0.0648 & 0.0189 & 3.4300 & 0.0010 & 0.0278 & 0.1019 & 0.1181 \\
\hline $\mathrm{R} 60$ & $\mathrm{R} 60.0$ & Localized edema & 61977 & 0.0741 & 0.0128 & 5.7900 & 0.0000 & 0.0490 & 0.0992 & 0.1161 \\
\hline C61 & C61. & Malignant neoplasm of prostate & 19707 & 0.0974 & 0.0262 & 3.7100 & 0.0000 & 0.0460 & 0.1489 & 0.1106 \\
\hline $\mathrm{H} 54$ & $\mathrm{H} 54.7$ & Unspecified visual loss & 25803 & 0.0907 & 0.0203 & 4.4800 & 0.0000 & 0.0510 & 0.1303 & 0.1098 \\
\hline K74 & K74.60 & Unspecified cirrhosis of liver & 8439 & 0.1148 & 0.0497 & 2.3100 & 0.0210 & 0.0175 & 0.2122 & 0.1076 \\
\hline D63 & D63.1 & Anemia in chronic kidney disease & 14823 & 0.0421 & 0.0124 & 3.3900 & 0.0010 & 0.0177 & 0.0665 & 0.1073 \\
\hline $\mathrm{N} 47$ & N47.6 & Balanoposthitis & 6273 & 0.0979 & 0.0421 & 2.3300 & 0.0200 & 0.0155 & 0.1804 & 0.1054 \\
\hline M10 & M10.9 & Gout, unspecified & 32778 & 0.1014 & 0.0223 & 4.5400 & 0.0000 & 0.0576 & 0.1452 & 0.1035 \\
\hline 167 & 167.89 & Other cerebrovascular disease & 16692 & 0.0534 & 0.0157 & 3.4000 & 0.0010 & 0.0226 & 0.0842 & 0.1021 \\
\hline 170 & 170.0 & Atherosclerosis of aorta & 19489 & 0.0537 & 0.0147 & 3.6400 & 0.0000 & 0.0248 & 0.0825 & 0.1000 \\
\hline C44 & C44.91 & Basal cell carcinoma of skin, unspecified & 21770 & 0.0769 & 0.0250 & 3.0800 & 0.0020 & 0.0280 & 0.1259 & 0.0906 \\
\hline 163 & 163.9 & Cerebral infarction, unspecified & 18075 & 0.0507 & 0.0173 & 2.9400 & 0.0030 & 0.0169 & 0.0845 & 0.0905 \\
\hline T82 & T82.818A & $\begin{array}{l}\text { Embolism due to vascular prosth dev/grft, } \\
\text { initial encounter }\end{array}$ & 6593 & 0.0462 & 0.0232 & 1.9900 & 0.0470 & 0.0006 & 0.0917 & 0.0866 \\
\hline M15 & M15.9 & Polyosteoarthritis, unspecified & 70950 & 0.0799 & 0.0191 & 4.1800 & 0.0000 & 0.0424 & 0.1173 & 0.0826 \\
\hline J18 & $\mathrm{J} 18.9$ & Pneumonia, unspecified organism & 32422 & 0.0512 & 0.0155 & 3.3000 & 0.0010 & 0.0208 & 0.0817 & 0.0796 \\
\hline Z65 & Z65.9 & $\begin{array}{l}\text { Problem related to unspecified } \\
\text { psychosocial circumstances }\end{array}$ & 127694 & 0.0833 & 0.0209 & 3.9800 & 0.0000 & 0.0423 & 0.1243 & 0.0730 \\
\hline 165 & 165.29 & $\begin{array}{l}\text { Occlusion and stenosis of unspecified } \\
\text { carotid artery }\end{array}$ & 16848 & 0.0413 & 0.0162 & 2.5500 & 0.0110 & 0.0096 & 0.0729 & 0.0717 \\
\hline R01 & R01.1 & Cardiac murmur, unspecified & 14466 & 0.0708 & 0.0309 & 2.2900 & 0.0220 & 0.0102 & 0.1313 & 0.0715 \\
\hline R33 & R33.9 & Retention of urine, unspecified & 20323 & 0.0408 & 0.0164 & 2.4900 & 0.0130 & 0.0086 & 0.0729 & 0.0694 \\
\hline
\end{tabular}




\begin{tabular}{|c|c|c|c|c|c|c|c|c|c|c|}
\hline R32 & R32. & Unspecified urinary incontinence & 18819 & 0.0441 & 0.0192 & 2.2900 & 0.0220 & 0.0064 & 0.0817 & 0.0650 \\
\hline $\mathrm{R} 80$ & $\mathrm{R} 80.3$ & Bence Jones proteinuria & 13651 & 0.0517 & 0.0262 & 1.9700 & 0.0490 & 0.0003 & 0.1030 & 0.0646 \\
\hline N17 & N17.9 & Acute kidney failure, unspecified & 31785 & 0.0282 & 0.0123 & 2.2900 & 0.0220 & 0.0041 & 0.0523 & 0.0604 \\
\hline R97 & R97.2 & Elevated prostate specific antigen [PSA] & 36803 & 0.0617 & 0.0248 & 2.4900 & 0.0130 & 0.0131 & 0.1103 & 0.0598 \\
\hline $\mathrm{H} 02$ & $\mathrm{H} 02.839$ & $\begin{array}{l}\text { Dermatochalasis of unspecified eye, } \\
\text { unspecified eyelid }\end{array}$ & 29211 & 0.0526 & 0.0216 & 2.4300 & 0.0150 & 0.0102 & 0.0950 & 0.0580 \\
\hline L57 & L57.0 & Actinic keratosis & 46813 & 0.0494 & 0.0226 & 2.1900 & 0.0290 & 0.0051 & 0.0937 & 0.0505 \\
\hline E87 & E87.6 & Hypokalemia & 54353 & 0.0312 & 0.0138 & 2.2600 & 0.0240 & 0.0041 & 0.0583 & 0.0492 \\
\hline R91 & R91.8 & $\begin{array}{l}\text { Other nonspecific abnormal finding of lung } \\
\text { field }\end{array}$ & 37585 & -0.0542 & 0.0251 & -2.1600 & 0.0310 & -0.1035 & -0.0049 & -0.0523 \\
\hline 151 & 151.7 & Cardiomegaly & 24480 & -0.0507 & 0.0225 & -2.2500 & 0.0240 & -0.0948 & -0.0066 & -0.0592 \\
\hline R31 & R31.9 & Hematuria, unspecified & 42562 & -0.0824 & 0.0309 & -2.6700 & 0.0080 & -0.1430 & -0.0219 & -0.0600 \\
\hline R78 & R78.89 & $\begin{array}{l}\text { Finding of oth substances, not normally } \\
\text { found in blood }\end{array}$ & 32503 & -0.0692 & 0.0268 & -2.5800 & 0.0100 & -0.1217 & -0.0166 & -0.0624 \\
\hline Z09 & Z09. & $\begin{array}{l}\text { Encntr for } \mathrm{f} / \mathrm{u} \text { exam aft trtmt for cond oth } \\
\text { than malig neoplm }\end{array}$ & 55214 & -0.0987 & 0.0319 & -3.0900 & 0.0020 & -0.1612 & -0.0361 & -0.0660 \\
\hline $\mathrm{R} 42$ & R42. & Dizziness and giddiness & 67634 & -0.0950 & 0.0282 & -3.3700 & 0.0010 & -0.1503 & -0.0398 & -0.0677 \\
\hline R55 & R55. & Syncope and collapse & 30812 & -0.0755 & 0.0277 & -2.7200 & 0.0070 & -0.1298 & -0.0211 & -0.0680 \\
\hline M62 & M62.81 & Muscle weakness (generalized) & 71400 & -0.0695 & 0.0206 & -3.3700 & 0.0010 & -0.1099 & -0.0291 & -0.0696 \\
\hline Z86 & Z86.010 & Personal history of colonic polyps & 103774 & -0.0745 & 0.0197 & -3.7700 & 0.0000 & -0.1132 & -0.0358 & -0.0697 \\
\hline N13 & N13.30 & Unspecified hydronephrosis & 13082 & -0.0708 & 0.0338 & -2.1000 & 0.0360 & -0.1369 & -0.0046 & -0.0727 \\
\hline 112 & 112.9 & $\begin{array}{l}\text { Hypertensive chronic kidney disease w stg } \\
1-4 / \text { unsp chr kdny }\end{array}$ & 26422 & -0.0329 & 0.0131 & -2.5200 & 0.0120 & -0.0585 & -0.0073 & -0.0762 \\
\hline E83 & E83.42 & Hypomagnesemia & 22641 & -0.0670 & 0.0234 & -2.8700 & 0.0040 & -0.1128 & -0.0212 & -0.0792 \\
\hline L03 & L03.90 & Cellulitis, unspecified & 70833 & -0.1097 & 0.0282 & -3.8900 & 0.0000 & -0.1651 & -0.0544 & -0.0797 \\
\hline L98 & L98.9 & $\begin{array}{l}\text { Disorder of the skin and subcutaneous } \\
\text { tissue, unspecified }\end{array}$ & 55257 & -0.1296 & 0.0345 & -3.7600 & 0.0000 & -0.1971 & -0.0621 & -0.0801 \\
\hline F06 & F06.30 & $\begin{array}{l}\text { Mood disorder due to known physiological } \\
\text { condition, unsp }\end{array}$ & 31408 & -0.0931 & 0.0291 & -3.2000 & 0.0010 & -0.1502 & -0.0360 & -0.0816 \\
\hline G93 & G93.3 & Postviral fatigue syndrome & 39656 & -0.0957 & 0.0253 & -3.7800 & 0.0000 & -0.1453 & -0.0461 & -0.0881 \\
\hline E86 & E86.0 & Dehydration & 28085 & -0.0714 & 0.0210 & -3.4000 & 0.0010 & -0.1126 & -0.0302 & -0.0899 \\
\hline Z79 & Z79.899 & Other long term (current) drug therapy & 147374 & -0.0736 & 0.0162 & -4.5500 & 0.0000 & -0.1054 & -0.0419 & -0.0915 \\
\hline D50 & D50.9 & Iron deficiency anemia, unspecified & 31287 & -0.0753 & 0.0208 & -3.6200 & 0.0000 & -0.1160 & -0.0345 & -0.0919 \\
\hline Z91 & Z91.19 & $\begin{array}{l}\text { Patient's noncompliance w oth medical } \\
\text { treatment and regimen }\end{array}$ & 64332 & -0.1130 & 0.0250 & -4.5200 & 0.0000 & -0.1620 & -0.0640 & -0.0932 \\
\hline K56 & K56.60 & Unspecified intestinal obstruction & 8535 & -0.0953 & 0.0406 & -2.3500 & 0.0190 & -0.1748 & -0.0159 & -0.0966 \\
\hline M20 & M20.40 & $\begin{array}{l}\text { Other hammer toe(s) (acquired), } \\
\text { unspecified foot }\end{array}$ & 43692 & -0.1552 & 0.0345 & -4.5000 & 0.0000 & -0.2228 & -0.0875 & -0.1006 \\
\hline 147 & 147.1 & Supraventricular tachycardia & 12549 & -0.1055 & 0.0346 & -3.0500 & 0.0020 & -0.1732 & -0.0377 & -0.1061 \\
\hline M19 & M19.90 & Unspecified osteoarthritis, unspecified site & 89625 & -0.1818 & 0.0320 & -5.6800 & 0.0000 & -0.2445 & -0.1190 & -0.1095 \\
\hline B95 & B95.61 & $\begin{array}{l}\text { Methicillin suscep staph infct causing dis } \\
\text { classd elswhr }\end{array}$ & 13437 & -0.1003 & 0.0321 & -3.1300 & 0.0020 & -0.1631 & -0.0374 & -0.1130 \\
\hline $\mathrm{R} 25$ & $\mathrm{R} 25.2$ & Cramp and spasm & 28206 & -0.1680 & 0.0389 & -4.3200 & 0.0000 & -0.2443 & -0.0918 & -0.1133 \\
\hline
\end{tabular}




\begin{tabular}{|c|c|c|c|c|c|c|c|c|c|c|}
\hline F05 & F05. & $\begin{array}{l}\text { Delirium due to known physiological } \\
\text { condition }\end{array}$ & 3612 & -0.0458 & 0.0203 & -2.2600 & 0.0240 & -0.0855 & -0.0061 & -0.1148 \\
\hline J98 & J98.4 & Other disorders of lung & 35483 & -0.1159 & 0.0247 & -4.6800 & 0.0000 & -0.1644 & -0.0674 & -0.1154 \\
\hline S22 & S22.39XA & $\begin{array}{l}\text { Fracture of one rib, unsp side, init for clos } \\
f x\end{array}$ & 10436 & -0.1438 & 0.0473 & -3.0400 & 0.0020 & -0.2366 & -0.0511 & -0.1165 \\
\hline K31 & K31.89 & Other diseases of stomach and duodenum & 15241 & -0.1706 & 0.0496 & -3.4400 & 0.0010 & -0.2679 & -0.0734 & -0.1169 \\
\hline G46 & G46.4 & Cerebellar stroke syndrome & 4838 & -0.0887 & 0.0379 & -2.3400 & 0.0190 & -0.1631 & -0.0144 & -0.1205 \\
\hline 111 & 111.9 & $\begin{array}{l}\text { Hypertensive heart disease without heart } \\
\text { failure }\end{array}$ & 28332 & -0.0920 & 0.0190 & -4.8500 & 0.0000 & -0.1291 & -0.0548 & -0.1219 \\
\hline 113 & 113.0 & $\begin{array}{l}\text { Hyp hrt \& chr kdny dis w hrt fail and stg 1- } \\
\text { 4/unsp chr kdny }\end{array}$ & 9823 & -0.0454 & 0.0133 & -3.4100 & 0.0010 & -0.0716 & -0.0193 & -0.1239 \\
\hline Z92 & Z92.3 & Personal history of irradiation & 8961 & -0.1416 & 0.0468 & -3.0200 & 0.0030 & -0.2334 & -0.0498 & -0.1287 \\
\hline $\mathrm{H} 43$ & $\mathrm{H} 43.819$ & Vitreous degeneration, unspecified eye & 38102 & -0.1599 & 0.0280 & -5.7000 & 0.0000 & -0.2148 & -0.1049 & -0.1304 \\
\hline Z48 & Z48.02 & Encounter for removal of sutures & 78659 & -0.1961 & 0.0303 & -6.4800 & 0.0000 & -0.2554 & -0.1368 & -0.1306 \\
\hline G81 & G81.90 & $\begin{array}{l}\text { Hemiplegia, unspecified affecting } \\
\text { unspecified side }\end{array}$ & 3445 & -0.0999 & 0.0456 & -2.1900 & 0.0280 & -0.1893 & -0.0105 & -0.1327 \\
\hline Y83 & Y83.1 & $\begin{array}{l}\text { Implnt of artif int dev cause abn } \\
\text { react/compl, w/o misadvnt }\end{array}$ & 11053 & -0.1500 & 0.0426 & -3.5200 & 0.0000 & -0.2335 & -0.0666 & -0.1394 \\
\hline D62 & D62. & Acute posthemorrhagic anemia & 6406 & -0.1002 & 0.0306 & -3.2800 & 0.0010 & -0.1601 & -0.0403 & -0.1441 \\
\hline 120 & 120.8 & Other forms of angina pectoris & 31188 & -0.1400 & 0.0241 & -5.8100 & 0.0000 & -0.1872 & -0.0928 & -0.1445 \\
\hline R06 & R06.02 & Shortness of breath & 118999 & -0.1827 & 0.0247 & -7.3900 & 0.0000 & -0.2311 & -0.1342 & -0.1459 \\
\hline G62 & G62.9 & Polyneuropathy, unspecified & 14108 & -0.1832 & 0.0409 & -4.4800 & 0.0000 & -0.2635 & -0.1030 & -0.1496 \\
\hline Z13 & Z13.6 & $\begin{array}{l}\text { Encounter for screening for cardiovascular } \\
\text { disorders }\end{array}$ & 237211 & -0.1886 & 0.0286 & -6.5900 & 0.0000 & -0.2447 & -0.1325 & -0.1519 \\
\hline S91 & S91.309A & $\begin{array}{l}\text { Unspecified open wound, unspecified foot, } \\
\text { initial encounter }\end{array}$ & 11184 & -0.1519 & 0.0374 & -4.0600 & 0.0000 & -0.2253 & -0.0786 & -0.1587 \\
\hline R39 & R39.15 & Urgency of urination & 29413 & -0.2332 & 0.0372 & -6.2700 & 0.0000 & -0.3061 & -0.1604 & -0.1648 \\
\hline Z98 & Z98.890 & Other specified postprocedural states & 92911 & -0.2348 & 0.0272 & -8.6400 & 0.0000 & -0.2881 & -0.1815 & -0.1663 \\
\hline T83 & T83.098A & $\begin{array}{l}\text { Mech compl of other urinary catheter, initial } \\
\text { encounter }\end{array}$ & 3957 & -0.1205 & 0.0408 & -2.9500 & 0.0030 & -0.2004 & -0.0406 & -0.1685 \\
\hline H52 & H52.4 & Presbyopia & 192666 & -0.1513 & 0.0212 & -7.1400 & 0.0000 & -0.1928 & -0.1097 & -0.1731 \\
\hline 116 & 116.0 & Hypertensive urgency & 5096 & -0.2349 & 0.0548 & -4.2900 & 0.0000 & -0.3423 & -0.1275 & -0.2227 \\
\hline J16 & $\mathrm{J} 16.8$ & $\begin{array}{l}\text { Pneumonia due to other specified } \\
\text { infectious organisms }\end{array}$ & 1240 & -0.1815 & 0.0750 & -2.4200 & 0.0160 & -0.3285 & -0.0345 & -0.2321 \\
\hline Z23 & Z23. & Encounter for immunization & 260232 & -0.3022 & 0.0320 & -9.4500 & 0.0000 & -0.3649 & -0.2395 & -0.2325 \\
\hline G21 & G21.11 & Neuroleptic induced parkinsonism & 2043 & -0.1384 & 0.0412 & -3.3600 & 0.0010 & -0.2193 & -0.0576 & -0.2494 \\
\hline 197 & 197.710 & $\begin{array}{l}\text { Intraoperative cardiac arrest during cardiac } \\
\text { surgery }\end{array}$ & 2682 & -0.2555 & 0.0660 & -3.8700 & 0.0000 & -0.3849 & -0.1260 & -0.2650 \\
\hline Z16 & Z16.11 & Resistance to penicillins & 2546 & -0.2529 & 0.0682 & -3.7100 & 0.0000 & -0.3866 & -0.1192 & -0.2690 \\
\hline T87 & T87.89 & Other complications of amputation stump & 1707 & -0.2549 & 0.0842 & -3.0300 & 0.0020 & -0.4199 & -0.0899 & -0.2772 \\
\hline W05 & W05.0XXA & $\begin{array}{l}\text { Fall from non-moving wheelchair, initial } \\
\text { encounter }\end{array}$ & 660 & -0.1834 & 0.0706 & -2.6000 & 0.0090 & -0.3219 & -0.0450 & -0.3132 \\
\hline
\end{tabular}


medRxiv preprint doi: https://doi.org/10.1101/2022.01.22.22269694; this version posted February 21, 2022. The copyright holder for this preprint (which was not certified by peer review) is the author/funder, who has granted medRxiv a license to display the preprint in perpetuity.

This article is a US Government work. It is not subject to copyright under 17 USC 105 and is also made available for use under a CCO license.

COMORBIDITIES IN MODELS FOR COVID-19 DEATH

TABLE 3: COMPARISON OF METHODS FOR HANDLING COMORBIDITIES

\begin{tabular}{|l|c|c|c|}
\hline & Charleson & Elixhauser & P(Death)Dx \\
\hline Diagnosis-specific & No & No & Yes \\
\hline Includes all relevant diagnosis & No & No & Yes \\
\hline Number of comorbidities & 19 & None & Actual risk \\
\hline Weighting & Arbitrary & Yes & Yes \\
\hline Includes protective factors & No & No & Yes \\
\hline Handles rare conditions & No & No & Yes \\
\hline $\begin{array}{l}\text { Effect of each comorbidity adjusted for } \\
\text { others }\end{array}$ & No & No & Yes \\
\hline $\begin{array}{l}\text { Identifies all comorbid patterns and } \\
\text { associated risks }\end{array}$ & No & Inpatient only & Inpatient/outpatient \\
\hline Setting of extracted diagnostic codes & Inpatient only & & \\
\hline
\end{tabular}


medRxiv preprint doi: https://doi.org/10.1101/2022.01.22.22269694; this version posted February 21, 2022. The copyright holder for this preprint

(which was not certified by peer review) is the author/funder, who has granted medRxiv a license to display the preprint in perpetuity.

This article is a US Government work. It is not subject to copyright under 17 USC 105 and is also made available for use under a CCO license.

COMORBIDITIES IN MODELS FOR COVID-19 DEATH

\section{REFERENCES}

1. King JT Jr, Yoon JS, Rentsch CT, et al. Development and validation of a 30-day mortality index based on pre-existing medical administrative data from 13,323 COVID-19 patients: The Veterans Health Administration COVID-19 (VACO) Index. PLoS One. 2020 Nov 11;15(11):e0241825. doi: 10.1371/journal.pone.0241825.

2. Ioannou GN, Green P, Fan VS, et al. "Development of COVIDVax model to estimate the risk of SARS-CoV-2-related death among 7.6 million US veterans for use in vaccination prioritization," JAMA Network Open 2021; 4:e214347.

3. Barda N, Riesel D, Akriv A, et a. Developing a COVID-19 mortality risk prediction model when individual-level data are not available. Nature Communications 2020. DOI: 10.1038/s41467-020-18297-9.

4. Hippisley-Cox J, Coupland CAC, Mehta N, et al. Risk prediction of covid-19 related death and hospital admission in adults after covid-19 vaccination: national prospective cohort study. BMJ 2021;374:n2244.

5. Cohn BA, Cirillo PM, Murphy CC, et al. SARS-CoV-2 vaccine protection and deaths among US veterans during 2021. Science 2021. DOI: 10.1126/science.abm0620.

6. Tuty Kuswardhani RA, Henrina J, Pranata R, Anthonius Lim M, Lawrensia S, Suastika K. Charlson comorbidity index and a composite of poor outcomes in COVID-19 patients: A systematic review and meta-analysis. Diabetes Metab Syndr. 2020 Nov-Dec;14(6):21032109. doi: 10.1016/j.dsx.2020.10.022. Epub 2020 Oct 28.PMID: 33161221.

7. Austin SR, Wong YN, Uzzo RG, Beck JR, Egleston BL. Why summary comorbidity measures such as the Charlson Comorbidity Index and Elixhauser Score work. Med Care. 2015 Sep;53(9):e65-72. doi: 10.1097/MLR.0b013e318297429c. 